\title{
EVALUACIÓN DEL FITOPLANCTON Y LA CALIDAD DEL AGUA DE UN ARROYO SUBTROPICAL DEL NOROESTE ARGENTINO
}

\author{
Evaluation of phytoplankton and water quality of a subtropical stream in the Northwest of \\ Argentina
}

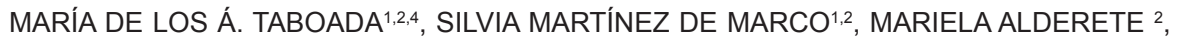 MARÍA L. GULTEMIRIAN ${ }^{2,3}$ \& BEATRIZ C. TRACANNA ${ }^{1,2,4}$

\begin{abstract}
Resumen: El arroyo Calimayo pertenece a la cuenca del río Salí, principal red fluvial de la región. Este sistema lótico fue conocido por su gran diversidad biológica. Debido a la instalación de una papelera y sus efluentes las condiciones ecológicas se modificaron. El objetivo fue analizar el fitoplancton y la calidad del agua del arroyo Calimayo. Se realizaron muestreos estacionales desde invierno/2012 hasta otoño/2013 en tres sitios (S1: tramo alto, S2 y S3: sectores medio y bajo, respectivamente). Se recolectaron muestras ambientales y biológicas según metodología convencional. Los mayores registros de temperatura, $\mathrm{pH}$ y conductividad eléctrica se presentaron en S2 y S3. EI S1 tuvo los máximos valores de oxígeno disuelto y la menor demanda bioquímica de oxígeno. Las diatomeas sobresalieron tanto cuali como cuantitativamente del resto de los grupos algales. El análisis de redundancia (RDA) permitió diferenciar dos grupos fitoplanctónicos en relación a variables abióticas. El S1 estuvo influenciado por estacionalidad, litología y fisicoquímica del agua, denotando un buen estado ecológico. En S2-S3 las condiciones ecológicas se vieron afectadas por el estado trófico del agua y los efluentes polutos, por lo que la calidad del agua y el fitoplancton se vieron notoriamente deteriorados. La ficoflora resultó ser un buen indicador de las condiciones ecológicas de este sistema lótico. Podemos afirmar que las condiciones limnológicas de este arroyo desde la década del 90 hasta la actualidad, fueron degradándose por la presencia de los efluentes industriales.
\end{abstract}

Palabras clave: Argentina, arroyo Calimayo, efluentes polutos, microalgas, Tucumán, variables abióticas y bióticas.

Summary: The Calimayo stream is part of the Salí river basin, the main fluvial network in the region. This lotic system was recognized for its great biodiversity. The settlement of a paper industry altered its ecological conditions due to the production of polluting effluents. The aim of this study was to analyze the phytoplankton of the Calimayo stream in relation to water quality. Sampling was done under a seasonal basis from Winter 2012 to Autumn 2013 at three sites S1, S2, and S3. Environmental and biological samples were collected following standard procedures. The highest values of temperature, $\mathrm{pH}$ and electric conductivity were registered in sites S2 and S3. Site S1 had the maximum values of dissolved oxygen and the lower biochemical oxygen demand. Diatoms excelled qualitatively and quantitatively among the other algal groups. Redundancy analysis (RDA) allowed to differentiate two phytoplanktonic groups related to abiotic variables. Site S1 was influenced by seasonality, relief, lithology and physicochemistry of water, denoting good ecological conditions. At S2 and S3 the phycoflora was affected by the trophic state of the water and the pollutants, which caused a notable deterioration of water quality

\footnotetext{
${ }^{1}$ Instituto de Ficología, Fund. M. Lillo. Miguel Lillo 251, T4000JFE- San Miguel de Tucumán, Argentina.

${ }^{2}$ Instituto de Limnología del Noroeste Argentino-Fac. de Ciencias Naturales e IML, Univ. Nac. De Tucumán. Miguel Lillo 205, T4000JFE- San Miguel de Tucumán, Argentina.

${ }^{3}$ Instituto de Biología Neotropical- CONICET.

${ }^{4}$ Unidad Ejecutora Lillo-CONICET. E-mail: mtaboada@lillo.org.ar
} 
and phytoplankton. Phytoplankton resulted to be a good indicator of the ecological conditions of this lotic system. We can affirm that the limnological conditions of the Calimayo stream from the 90 s to the present have been degraded to the presence of industrial effluents.

Key words: Abiotic and biotic variables, Argentina, Calimayo stream, microalgae, polluted effluents, Tucumán.

\section{Introducción}

Las aguas continentales varían enormemente en su contenido y composición mineral, principalmente debido a la litología, el clima y la vegetación. En las últimas décadas los ecosistemas acuáticos presentaron profundas modificaciones como producto de un aumento en la generación de residuos sólidos y líquidos debido a las actividades antrópicas (Western, 2001; Dolbeth et al., 2003; Fontúrbel, 2005). La degradación de los ambientes lóticos no sólo limita la disponibilidad de agua para el consumo humano, también afecta a los ciclos biogeoquímicos a nivel global, por lo que es conveniente evaluar integralmente la salud ecológica de estos sistemas (Bojorge García \& Cantoral Uriza, 2016).

Los sistemas hídricos albergan una gran cantidad de organismos, por lo que los impactos como la contaminación inducen a cambios en la estructura de las taxocenosis, la función biológica de los sistemas y de los propios individuos. Tradicionalmente la valoración de los cursos fluviales se ha realizado en base a las características fisicoquímicas, sin embargo en la actualidad varios países han complementado sus evaluaciones mediante el uso de indicadores biológicos (Toro et al., 2003). Las variables abióticas aportan información referente a las posibles causas de la contaminación, mientras que la biota brinda una visión holística de los efectos a mediano y largo plazo (Hutson \& Roberts, 1990). Las algas constituyen una taxocenosis ecológicamente relevante, ya que contribuyen en los procesos físicos, químicos y biológicos de los ríos y arroyos (Battin et al., 2003). Además son importantes por ser bioindicadores, principalmente por su tolerancia y sensibilidad a los cambios ambientales, y por sus ciclos de vida reducidos (Licursi \& Goméz, 2013). Para obtener una evaluación global e integradora del sistema acuático se necesitan indicadores efectivos que sean aplicables en diversos ecosistemas hídricos y que respondan a los cambios tanto a escala espacial como temporal (Burns \& Ryder, 2001). El fitoplancton reúne los atributos necesarios para el biomonitoreo, ya que ha sido ampliamente utilizado a través de diversos parámetros: abundancia, diversidad específica, riqueza e índices de saprobiedad, de este modo el fitoplancton provee una medida directa de los impactos sobre el ecosistema (Seeligmann, 2000). Por lo que su estudio permite complementar un manejo sostenible de los sistemas lóticos (Bojorge García \& Cantoral Uriza, 2016).

Las características físicas de gran parte de la provincia de Tucumán, ubicada en el noroeste argentino, proporcionan un marco apropiado para la existencia de numerosos sistemas hídricos. El arroyo Calimayo forma parte de la cuenca del río Salí, principal red fluvial de la región. Esta red fluvial fue conocida por su riqueza en especies vegetales y animales (Miquelarena et al., 1990). A mediados de 1990 se instaló en la zona una fábrica papelera y las condiciones ecológicas de este sistema comenzaron a deteriorarse paulatinamente. Esta industria utiliza el bagazo de la caña de azúcar como materia prima, siendo los principales componentes: $25-45 \%$ de celulosa, 25-50\% de hemicelulosa y 10$30 \%$ de lignina (Antolín \& Oliva, 2003). En la manufactura se emplea sulfuro de sodio para la deslignificación de la fibra vegetal e hipoclorito de sodio para su blanqueo. La fábrica produce aproximadamente $120.000 \mathrm{tn} / \mathrm{año}$ de papel y su actividad es continua a lo largo del año. Cabe resaltar que esta industria cuenta con planta de tratamientos de efluentes, sin embargo no se encuentra operativa, de manera que se vierten los poluentes directamente por un canal de descarga que llega al arroyo. La gran cantidad de materia orgánica y nutrientes aportados pueden producir eutrofización, incremento de la conductividad eléctrica, alteración de la composición microbiológica y por consiguiente modificación en la calidad 
del agua (Karrash et al., 2006). El objetivo del presente trabajo fue analizar espacial y temporalmente el fitoplancton y la calidad del agua del arroyo Calimayo en relación a efluentes polutos provenientes de una papelera.

\section{Materiales y Métodos}

\section{Área de estudio}

El arroyo Calimayo se localiza en el departamento Lules (Tucumán-Argentina) y se origina en el Alto de Yerba Huasi (Sierras Centrales). Forma parte de la cuenca del río Colorado y tiene un régimen permanente, posee un área de aproximadamente $452 \mathrm{~km}^{2}$ hasta su desembocadura en el río Salí en su extremo oriental. La litología se constituye de sedimentos limo-arcillosos. El tipo de suelo pertenece al orden Molisol, en menor medida al orden Entisol y el clima es subtropical con lluvias estivales (Fernández, 2012). Los mayores registros pluviométricos se presentan en los meses de enero-febrero y el mínimo en julio. Los valores más elevados de temperatura ocurren de octubre a marzo, con su máximo en enero (Fernández, 1981). El tramo alto forma parte del bosque de transición ubicado entre los 400-700 m s.n.m., entre la llanura tucumana hacia el oriente y las cadenas montañosas al oeste, presenta un relieve de colinas con inclinación hacia el este y sudeste (Guido \& Sesma, 2014). En este sector, el arroyo presenta un sustrato pedregoso y un bosque de ribera bien desarrollado. Los representantes arbóreos destacados corresponden a: Blepharocalyx salicifolius (Kunth) O. Berg (horco molle) y especies exóticas como las moreras (Morus sp.). En los sectores medio y bajo, debido a la descarga de la industria las condiciones naturales se ven modificadas: el agua tiene color marrón, con bagazo, mucha espuma blanca en superficie y olor nauseabundo. Cercano al cuerpo de agua se destaca la presencia de amplios cultivos de caña de azúcar, un estrato arbóreo con algunos representantes de Salix spp. (sauce) y uno herbáceo constituido principalmente por Ricinus communis L. (tártago), especie exótica característica de ambientes disturbados y el helecho Equisetum giganteum L. (cola de caballo).

\section{Muestreo}

Se realizaron muestreos estacionales desde agosto de 2012 a mayo de 2013 (invierno/2012, primavera/2012, verano/2013 y otoño/2013) en tres sitios: $\mathrm{S} 1\left(26^{\circ} 55^{\prime} 55^{\prime \prime} \mathrm{S}, 6^{\circ} 23^{\prime} 17^{\prime \prime} \mathrm{O}\right.$, 482 m s.n.m.), ubicado en el tramo alto (aguas arriba de la descarga de efluentes polutos); S2 (26 $57^{\prime} 05^{\prime \prime} \mathrm{S}, 6^{\circ} 21^{\prime} 30^{\prime \prime} \mathrm{O}, 393$ m s.n.m.) y

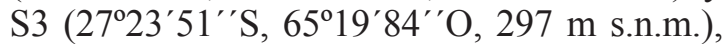
corresponden a los sectores medio y bajo (aguas abajo de la descarga), respectivamente (Fig. 1).

\section{Análisis fisicoquímicos y biológicos}

Para los parámetros físicos y químicos se empleó un analizador multiparamétrico digital portátil Water Quality Metre 850081, Sper Scientific, se midieron in situ: temperatura del agua, $\mathrm{pH}$ y conductividad eléctrica (CE). Asimismo, para la cuantificación del oxígeno disuelto (OD) se recolectó agua en frascos específicos y se fijó la muestra in situ según el método de Winkler, la muestra fue conservada a $-4^{\circ} \mathrm{C}$ hasta su análisis (APHA, 2005). En el laboratorio se analizaron también: iones mayoritarios, demanda bioquímica de oxígeno $\left(\mathrm{DBO}_{5}\right)$, bacterias coliformes totales, compuestos nitrogenados y ortofosfato. En la Tabla 1 se presentan las técnicas empleadas según APHA (2005). El control y aseguramiento de la calidad de los procedimientos de toma de muestras (las que se tomaron por duplicado en cada sitio), así como también las determinaciones analíticas en laboratorio, se realizaron de acuerdo a normas IRAM (290122-1996, 29012-3-1998, 301/ISO17025-2005).

La evaluación del tipo de agua se hizo mediante Diagrama de Piper-Hill-Langelier utilizando el programa RockWorks 15.

En cada sitio se recolectaron una muestra cualitativa y dos cuantitativas del fitoplancton. La muestra cualitativa fue extraída con una red de plancton de 20 micrones de malla que fue ubicada a favor de la corriente durante 20 minutos, el filtrado obtenido fue conservado en frascos. Para los análisis cuantitativos se recogió agua directamente en frascos plásticos de $250 \mathrm{cc}$. Ambas muestras fueron fijadas in situ con formaldehído al $4 \%$.

Parala obtención dela clorofila( $\mu$ gdeclorofila $a / \mathrm{L})$ se siguió el método monocromático de 


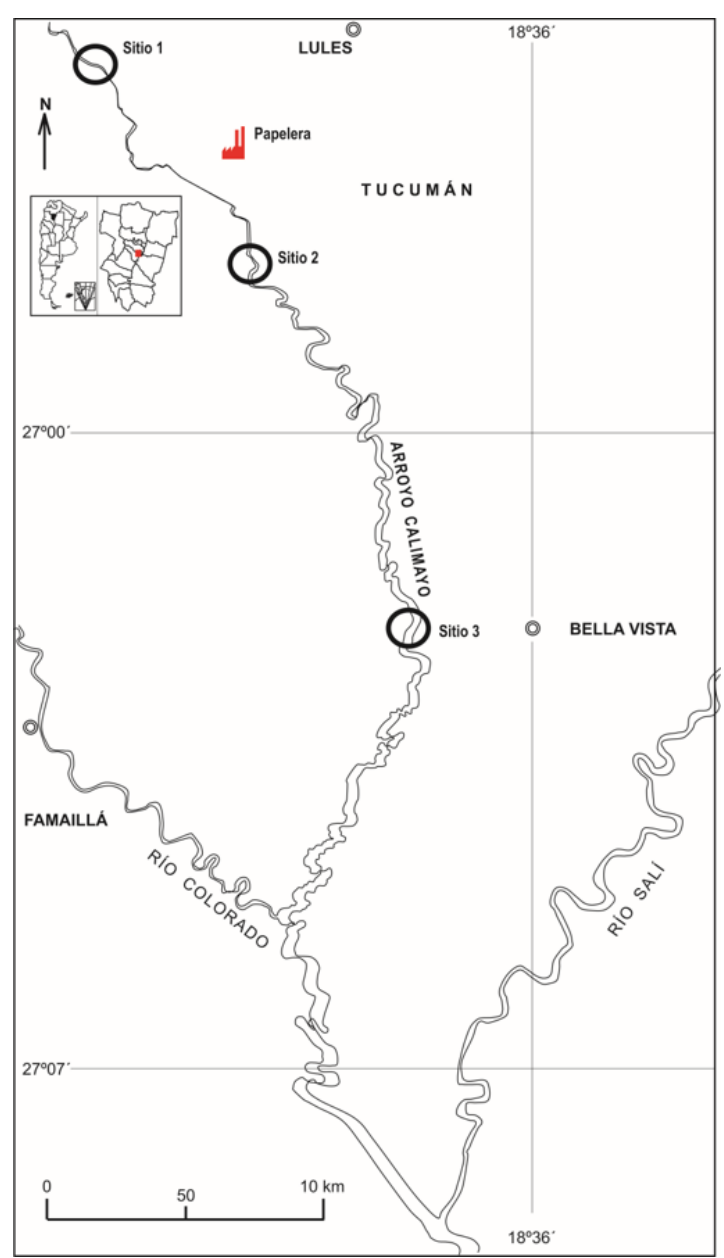

Fig. 1. Mapa de ubicación de los sitios muestreados y de la industria papelera.

Fig. 1. Location map of the sampled sites and the paper industry.

Loéz (1995). En laboratorio, el agua recogida (se tomaron 5 lt en cada sitio) fue filtrada, empleando filtros Whatman GF/C de acuerdo a lo recomendado por Holm-Hansen (1978) por medio de un aspirador a diafragma ECAM Aspirex. Para completar la lisis celular se procedió a congelar los filtros a $-18^{\circ} \mathrm{C}$. Posteriormente los filtros fueron triturados y macerados en oscuridad, utilizando metanol como solvente, debido a su mayor poder de penetración en las membranas celulares de las algas. El extracto obtenido fue filtrado nuevamente y se realizó la lectura mediante un espectrofotómetro. La concentración de clorofila $a$ fue calculada según la fórmula de Talling \& Driver (Ros, 1979).

Los estudios cualitativos se realizaron mediante microscopio binocular Zeiss Axio Lab 1 con cámara fotográfica incorporada y con contraste de fase a un aumento de 1200x. Para las Bacillariophyceae (diatomeas), se procedió a la eliminación de la materia orgánica según la metodología de Battarbee (1986), mediante peróxido de hidrógeno y calor. En la realización de los preparados permanentes se utilizó Naphrax ${ }^{\circledR}$ como medio de montaje. Para las determinaciones específicas se consultaron para Cyanophyta a Frémy (1930), Geitler (1932), Desikachary (1959), Starmach (1966), Anagnostidis \& Komárek (1988) y Komárek \& Anagnostidis (2005). En el caso de las Chlorophyta se siguió a Krieger (1937), Prescott (1961), Uherkovich (1966), Komárková-Legnerová (1969), Prescott et al. (1972) y Tracanna (1982, 1985). Para los análisis taxonómicos de Bacillariophyceae se consultaron Germain (1981), Krammer \& Lange-Bertalot (1986, 1988, 1991, 2004), Martínez de Fabricius (1996), Seeligmann (2000), Metzeltin et al. (2005), Levkov (2009), Echazu (2012), Levkov et al. (2013), Maidana et al. (2008, 2011), Seeligmann \& Maidana (2003, 2013), González Achem et al. (2014), Maidana \& Seeligmann (2006, 2015), entre otros. En las Euglenophyta se siguió a HuberPestalozzi (1955) y Tell \& Conforti (1986) y en Dinophyta a Boltovskoy (1999).

Para la abundancia algal se realizaron recuentos bajo microscopio invertido Zeiss IDO2 a un aumento de 400x según la metodología de Utermöhl (1958).

Las muestras fitoplanctónicas recolectadas fueron incorporadas a la Colección Ficológica (LIL) de la Fundación Miguel Lillo (LIL 25.149 - 25.160).

Para estimar y comparar la diversidad fitoplanctónica entre las diferentes muestras, se utilizó el índice de Shannon-Weaver (Magurran, 2004). Se aplicó el índice de contaminación por materia orgánica (ICOMO) (Ramírez González \& Viña Vizcaíno, 1998) que se puede calcular con diferentes variables. Las seleccionadas en este trabajo correspondieron a: Demanda bioquímica de 
Tabla 1. Metodologías empleadas para la determinación de las variables ambientales y biológicas.

Table 1. Methodologies used for the determination of environmental and biological variables.

\begin{tabular}{|c|c|c|c|}
\hline Variables & & Unidades & \\
\hline Conductividad eléctrica & $\mu S / c m$ & Conductímetro & APHA (2005) \\
\hline $\mathrm{pH}$ & $\begin{array}{l}\text { Unidades } \\
\text { de } \mathrm{pH}\end{array}$ & Método potenciométrico & APHA (2005) \\
\hline Carbonato y Bicarbonato & $\mathrm{mg} / \mathrm{l}$ & Método volumétrico & APHA (2005) \\
\hline Sulfato & $\mathrm{mg} / \mathrm{l}$ & Nefelométrico & APHA (2005) \\
\hline Cloruro & $\mathrm{mg} / \mathrm{l}$ & Método de Mohr & APHA (2005) \\
\hline Sodio & $\mathrm{mg} / \mathrm{l}$ & Espectrofotómetro de llama & APHA (2005) \\
\hline Potasio & $\mathrm{mg} / \mathrm{l}$ & $\begin{array}{l}\text { Espectrofotómetro de llama } \\
\text { Fotometría de emisión }\end{array}$ & APHA (2005) \\
\hline Calcio & $\mathrm{mg} / \mathrm{l}$ & Volumetría & APHA (2005) \\
\hline Magnesio & $\mathrm{mg} / \mathrm{l}$ & Volumetría & APHA (2005) \\
\hline Amonio & $\mathrm{mg} / \mathrm{l}$ & Método colorimétrico & $\begin{array}{l}\text { Kits Espectrofotómetro } \\
\text { (AQAssay) }\end{array}$ \\
\hline Nitrato & $\mathrm{mg} / \mathrm{l}$ & Método colorimétrico & $\begin{array}{l}\text { Espectofotómetro } 540 \\
\mathrm{~nm} .\end{array}$ \\
\hline Nitrito & $\mathrm{mg} / \mathrm{l}$ & Método colorimétrico & $\begin{array}{l}\text { Kits Espectrofotométrico } \\
\text { (AQAssay) }\end{array}$ \\
\hline Fosfato & $\mathrm{mg} / \mathrm{l}$ & Método colorimétrico & Espectrofotómetro \\
\hline Oxígeno disuelto & $\mathrm{mg} / \mathrm{l}$ & Volumétrico & APHA (2005) \\
\hline $\begin{array}{l}\text { Demanda Bioquímica de } \\
\text { Oxígeno }\end{array}$ & $\mathrm{mg} / \mathrm{l}$ & $\begin{array}{l}\text { Diferencia entre OD inicial y OD } \\
\text { final }\end{array}$ & APHA (2005) \\
\hline Bacterias Coliformes totales & $\mathrm{NMP} / 100 \mathrm{ml}$ & $\begin{array}{c}\text { Técnica de Filtración por } \\
\text { Membrana }\end{array}$ & APHA (2005) \\
\hline
\end{tabular}

oxígeno $\left(\mathrm{DBO}_{5}\right)$, coliformes totales (reflejan fuentes diferentes de contaminación orgánica) y el porcentaje de saturación del oxígeno (a partir de la estimación del oxígeno disuelto), ya que indica la respuesta o capacidad ambiental del ecosistema ante ese tipo de polución. También se utilizó el índice de Equidad de Pielou (1966). Para llevar a cabo la caracterización de la salud biológica del agua se empleó el índice de Saprobiedad de Pantle \& Buck (1955).

Se realizó un análisis de redundancia (RDA) para relacionar la matriz de abundancia de especies con una matriz de variables ambientales y poder detectar si existen grupos taxonómicos influenciados por algunas variables abióticas. Se aplicó esta técnica dado que en un examen preliminar los datos mostraron que las longitudes de los gradientes abióticos fueron menores a 2 condición sugerida para el uso de un modelo con respuesta lineal como es el RDA (Ter Braak \& Smilauer, 1998; Legendre \& Legendre 2000). De un total de 46 especies se seleccionaron 27 extrayéndose aquellas que presentaron menos de 10 individuos durante todo el período estudiado por considerarlas poco frecuentes (menos del 5\% del total registrado) (Aragón \& Morales, 2003). Los parámetros ambientales utilizados fueron: temperatura, $\mathrm{pH}$, oxígeno disuelto, $\mathrm{DBO}_{5}$, $\mathrm{HCO}_{3}^{-}, \mathrm{CO}_{3}{ }^{2-}, \mathrm{Cl}^{-}, \mathrm{Mg}^{2+}, \mathrm{Ca}^{2+}, \mathrm{K}^{+}, \mathrm{NO}_{3}^{-} \mathrm{y}$ $\mathrm{SO}_{4}^{2-}$. Dado que la conductividad eléctrica presentó valores de correlación superior al $80 \%$ con $\mathrm{Cl}^{-}$y $\mathrm{Na}^{+}$, se decidió utilizar de las tres variables solamente el cloruro para evitar 
el efecto de multicolinealidad (Legendre \& Legendre, 2000). Se tuvo en cuenta que la cantidad total de los ejes de RDA integrasen juntos al menos un $50 \%$ de la varianza total de la información registrada. Además, se consideraron a las variables ambientales que se correlacionaron con al menos un $40 \%$ de algún eje RDA seleccionado para la explicación de la asociación entre las especies de la ficoflora y las variables ambientales. Los análisis fueron realizados a través de software R13.1 (R Development Core Team, 2005).

Considerando sólo las especies que aparecen en el RDA, se elaboró una curva de abundancia - dominancia (Whittaker, 1965, 1972) basada en el log de la abundancia relativa de las especies registradas en el total de muestras para cada sitio $\log P_{i}=\log \left(\frac{n_{i n}}{n_{t m}}\right)$. , donde $n_{i m}$ es la abundancia de la especie $\mathrm{i}$ en el sitio $\mathrm{m}$ y $n_{t m}$ es el total de individuos registrados en el sitio $m(m=1,2,3)$. Para cada sitio se ubican a las especies de mayor a menor abundancia relativa.

\section{Resultados}

\section{Variables ambientales}

Los datos de las variables abióticas se presentan en la Tabla 2.

La temperatura del agua fluctuó de $13^{\circ} \mathrm{C}(\mathrm{S} 1$ invierno/2012) a $27^{\circ} \mathrm{C}(\mathrm{S} 2$ primavera/2012), evidenciando un incremento de esta variable aguas abajo del vertido de la papelera. El pH del agua fue alcalino con registros que oscilaron entre 7,2-8,7, con valores inferiores en S2 y S3.

La CE varió entre $280 \mu \mathrm{S} . \mathrm{cm}^{-1}$ (S1 verano/2013) y $2572 \mu \mathrm{S} . c m^{-1}$ (S2 invierno/2012) (Fig. 2).

Según el diagrama de Piper-Hill-Langelier se clasificó a las aguas del sitio 1 como bicarbonatadas-cálcicas-magnésicas y en S2-S3 fueron de tipo cloruradas-sulfatadassódicas (Fig. 3).

La menor concentración de OD se presentó en los sitios: S3 (0,06 $\mathrm{mg} \mathrm{l}^{-1}$ desde invierno/2012 a otoño/2013) y en $\mathrm{S} 2$ (0,06 en inv/12), mientras que en $\mathrm{S} 1$ se observó, en todo el periodo estudiado

Tabla 2. Variables fisicoquímicas del arroyo Calimayo.

Table 2. Physicochemical variables of the Calimayo stream.

\begin{tabular}{|c|c|c|c|c|c|c|c|c|c|c|c|c|}
\hline & \multicolumn{3}{|c|}{ Inv/12 } & \multicolumn{3}{|c|}{ Prim/12 } & \multicolumn{3}{|c|}{ Ver/13 } & \multicolumn{3}{|c|}{ Oto/13 } \\
\hline & S1 & S2 & S3 & S1 & S2 & S3 & S1 & S2 & S3 & S1 & S2 & S3 \\
\hline Temperatura ${ }^{\circ} \mathrm{C}$ & 13 & 23 & 19 & 20 & 27 & 24 & 21 & 25 & 24 & 15 & 20 & 24 \\
\hline $\mathrm{pH}(\mathrm{upH})$ & 8,7 & 7,3 & 7,2 & 8,3 & 7,7 & 7,4 & 8,3 & 7,9 & 7,9 & 8,6 & 7,5 & 7,2 \\
\hline $\mathrm{CE} \mu \mathrm{S} . \mathrm{cm}^{-1}$ & 631 & 2572 & 2000 & 470 & 2030 & 1933 & 280 & 1620 & 1400 & 476 & 2250 & 1880 \\
\hline OD mg. $\mathrm{L}^{-1}$ & 9,2 & 0,06 & 0,06 & 7,1 & 0,6 & 0,06 & 7,9 & 1 & 0,06 & 9,5 & 0,5 & 0,06 \\
\hline $\mathrm{DBO}_{5} \mathrm{mg} \mathrm{L}^{-1}$ & 0,6 & 338,8 & 133,8 & 0,6 & 116,1 & 248 & 0,3 & 119 & 94 & 0,9 & 263,92 & 263 \\
\hline $\mathrm{HCO}_{3}^{-} \mathrm{mg}^{-\mathrm{L}^{-1}}$ & 213,5 & 793 & 579,5 & 195,2 & 610 & 579,5 & 103,7 & 433,1 & 433 & 183 & 707,6 & 610 \\
\hline $\mathrm{CO}_{3}^{-} \mathrm{mg}^{-\mathrm{L}^{-1}}$ & 108 & 0,1 & 0,1 & 48 & 0,1 & 0,1 & 24 & 0,1 & 0,1 & 72 & 0,1 & 0,1 \\
\hline $\mathrm{SO}_{4}^{2+} \mathrm{mg}^{2-\mathrm{L}^{-1}}$ & 5,76 & 192,12 & 144 & 1,5 & 100,8 & 78,2 & 9,6 & 110 & 72 & 2,8 & 96 & 67 \\
\hline $\mathrm{Cl}^{-} \mathrm{mg} \cdot \mathrm{L}^{-1}$ & 17,73 & 294,32 & 265,9 & 24,8 & 290,7 & 290,7 & 17,7 & 241 & 191,4 & 17,7 & 315,5 & 262 \\
\hline $\mathrm{Na}^{+} \mathrm{mg} \cdot \mathrm{L}^{-1}$ & 23 & 368 & 299 & 17 & 330 & 399,9 & 9 & 230 & 189,9 & 16,9 & 310 & 270 \\
\hline $\mathrm{K}^{+} \mathrm{mg} \cdot \mathrm{L}^{-1}$ & 3,9 & 46,8 & 35,8 & 5 & 65 & 33,9 & 3,9 & 12,8 & 12,8 & 3,9 & 23 & 19 \\
\hline $\mathrm{Ca}^{2+} \mathrm{mg} \cdot \mathrm{L}^{-1}$ & 80 & 134 & 80 & 39,9 & 33,4 & 60 & 26,6 & 73,4 & 66,6 & 46,6 & 113 & 100 \\
\hline $\mathrm{Mg}^{2+} \mathrm{mg} \cdot \mathrm{L}^{-1}$ & 15,6 & 15,6 & 28,2 & 20 & 15,1 & 36 & 12 & 12 & 15,9 & 20 & 20 & 20 \\
\hline $\mathrm{NO}_{3}^{-} \mathrm{mg} \cdot \mathrm{L}^{-1}$ & 2,21 & 1,53 & 1,12 & 2,24 & 1,25 & 8,75 & 1,34 & 1,08 & 2,17 & 0,53 & 0,63 & 0,59 \\
\hline $\mathrm{NO}_{2}^{-} \cdot \mathrm{mg} \cdot \mathrm{L}^{-1}$ & 0,01 & 0,03 & 0,1 & 0,02 & 0,02 & 0,03 & 0,07 & 0,29 & 0,24 & 0,01 & 0,07 & 0,06 \\
\hline $\mathrm{NH}_{4}^{+} \mathrm{mg} \cdot \mathrm{L}^{-1}$ & 0,01 & 0,44 & 0,55 & 0,06 & 0,67 & 1,44 & 0,07 & 0,54 & 0,5 & 0,01 & 0,42 & 0,75 \\
\hline $\mathrm{PO}^{3}{ }_{4} \mathrm{mg} \cdot \mathrm{L}^{-1}$ & 0,05 & 0,62 & 0,75 & 0,19 & 0,29 & 0,85 & 0,12 & 0,84 & 0,8 & 0,2 & 0,51 & 0,85 \\
\hline
\end{tabular}


la mayor cantidad, siendo el máximo de $9,5 \mathrm{mg} \mathrm{l}^{-1}$ en otoño/2013. La $\mathrm{DBO}_{5}$ tuvo valores de $0,3 \mathrm{mg}$ $\mathrm{l}^{-1}$ en $\mathrm{S} 1$ durante verano/2013 y de $338 \mathrm{mg} \mathrm{l}^{-1}$ en invierno/2012 en S2 (Fig. 4).

El ortofosfato osciló de $0,05 \mathrm{mg} \mathrm{l}^{-1}$ (S1 en invierno/2012) a $0,85 \mathrm{mg}^{-1}(\mathrm{~S} 3$ en primavera/2012). El mínimo registro de $\mathrm{NO}_{3}^{-}$fue de $0,53 \mathrm{mg} \mathrm{l}^{-1}$ (S1 en otoño/2013) y el máximo de $8,75 \mathrm{mg}^{-1}$ (S3 en primavera/2012). La concentración de $\mathrm{NO}_{2}^{-}$fluctuó de $0,01 \mathrm{mg} \mathrm{l}^{-1}(\mathrm{~S} 1$ invierno/2012) a $0,29 \mathrm{mg} \mathrm{l}^{-1}(\mathrm{~S} 2$ verano/2013). El mínimo contenido de $\mathrm{NH}_{4}^{+}$de $0,01 \mathrm{mg} \mathrm{l}^{-1}$ se observó en S1 invierno/2012 y el máximo fue $0,75 \mathrm{mg} \mathrm{l}^{-1}$ en S3 (otoño/2013). Los valores de los iones mayoritarios se presentan en la Tabla 2.

\section{Variables biológicas}

Se determinaron 80 taxones fitoplanctónicos, las diatomeas estuvieron representadas por 60 , las algas verdes por 9, las cianobacterias con 7 y los euglenoides con 4. En invierno/2012 la riqueza específica presentó el menor registro con 18 (S2) y el máximo con 62 (S1) (Tabla 3 y Fig. 5). Los géneros de diatomeas con mayor número de especies fueron: Gomphonema y Navicula (9) y Nitzschia (8). El valor del índice de diversidad osciló entre 1 en S2 (invierno/2012) y 4 en el sitio 1 para la primavera/2013, en general S1 presentó el doble de riqueza y valores mayores en todos los momentos registrados. La equidad varió entre 0,44 (S1 primavera/2012) y 1 (S2 invierno/2012) (Tabla 4).
El menor valor del ICOMO se observó en el sitio 1 para invierno/2013 (0.03), mientras que los sitios 2 y 3 siempre presentaron el máximo registro de 1. El índice de Saprobiedad osciló entre 1,7 (S1 verano/2013) y 3,7 (S2 otoño/2013) (Tabla 4).

La abundancia algal fluctúo entre $46 \mathrm{ind} / \mathrm{ml}$ en el sitio 3 (otoño/2013) y 359 ind $/ \mathrm{ml}$ en S1 (invierno/2012). Las diatomeas realizaron el mayor aporte a las densidades fitoplanctónicas, con valores comprendidos entre 11-334 ind $\mathrm{ml}^{-1}$ en $\mathrm{S} 2$ y $\mathrm{S} 1$ respectivamente, en invierno/2012. La menor abundancia de las algas verdeazuladas de 7 ind $\mathrm{ml}^{-1}$ se observó en S1 (primavera/2012) y la mayor de 63 ind $\mathrm{ml}^{-1}$ en S3 durante el verano/2013. La densidad de las algas verdes fluctuó de 7 (S1 verano/2013) a 35 ind $\mathrm{ml}^{-1}$ (S2 primavera/2012), los euglenoides solamente se registraron en S2 con 2 ind $\mathrm{ml}^{-1}$ en verano/2013 y 9 ind $\mathrm{ml}^{-1}$ en primavera/2012 (Fig. 6).

La concentración mínima de clorofila $a$ $\left(0,03 \mu \mathrm{g}^{-1}\right)$ se dio, en general, tanto en $\mathrm{S} 2$ como S3 para la mayoría de los meses analizados, mientras que la mayor $\left(4 \mu \mathrm{g} \mathrm{l}^{-1}\right) \mathrm{se}$ presentó en S1 durante el invierno/2012 (S1) (Fig. 7).

Al relacionarse las abundancias de las especies del fitoplancton con las variables ambientales mediante el RDA (Tabla 5 y Fig. 8) se observó que los dos primeros ejes canónicos acumularon un $54 \%$ de la proporción de

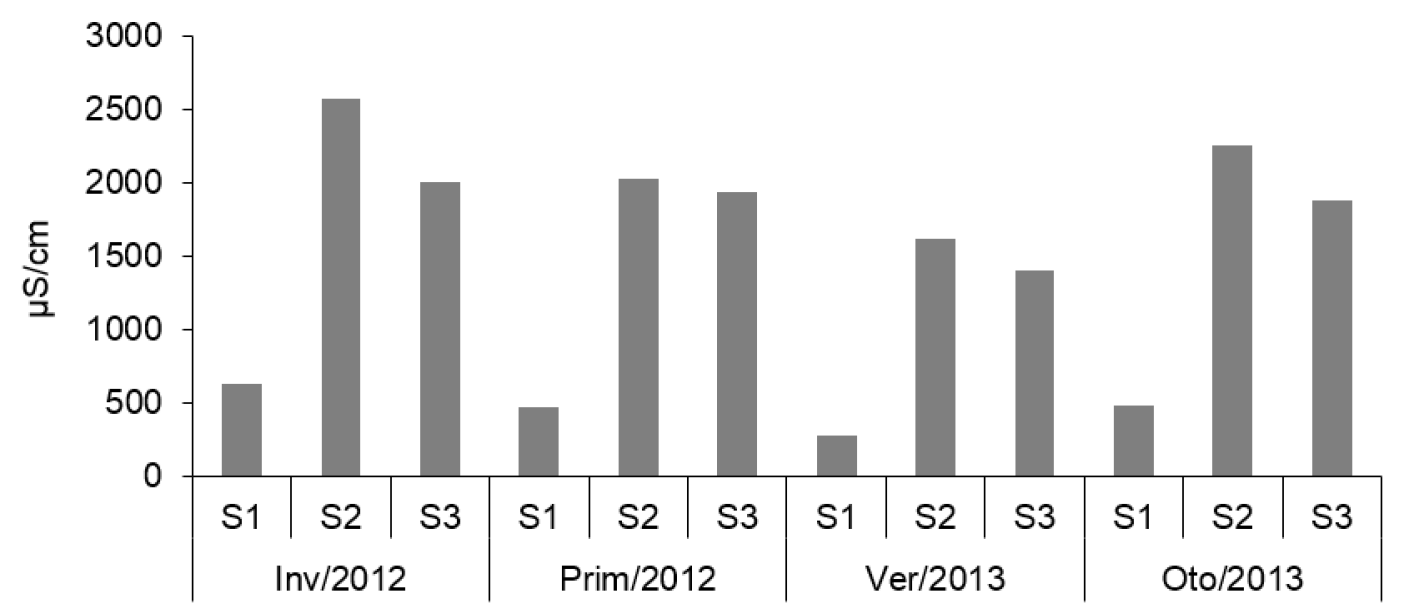

Fig. 2. Variación de la conductividad eléctrica durante el periodo analizado.

Fig. 2. Variation of electrical conductivity during the period analyzed. 


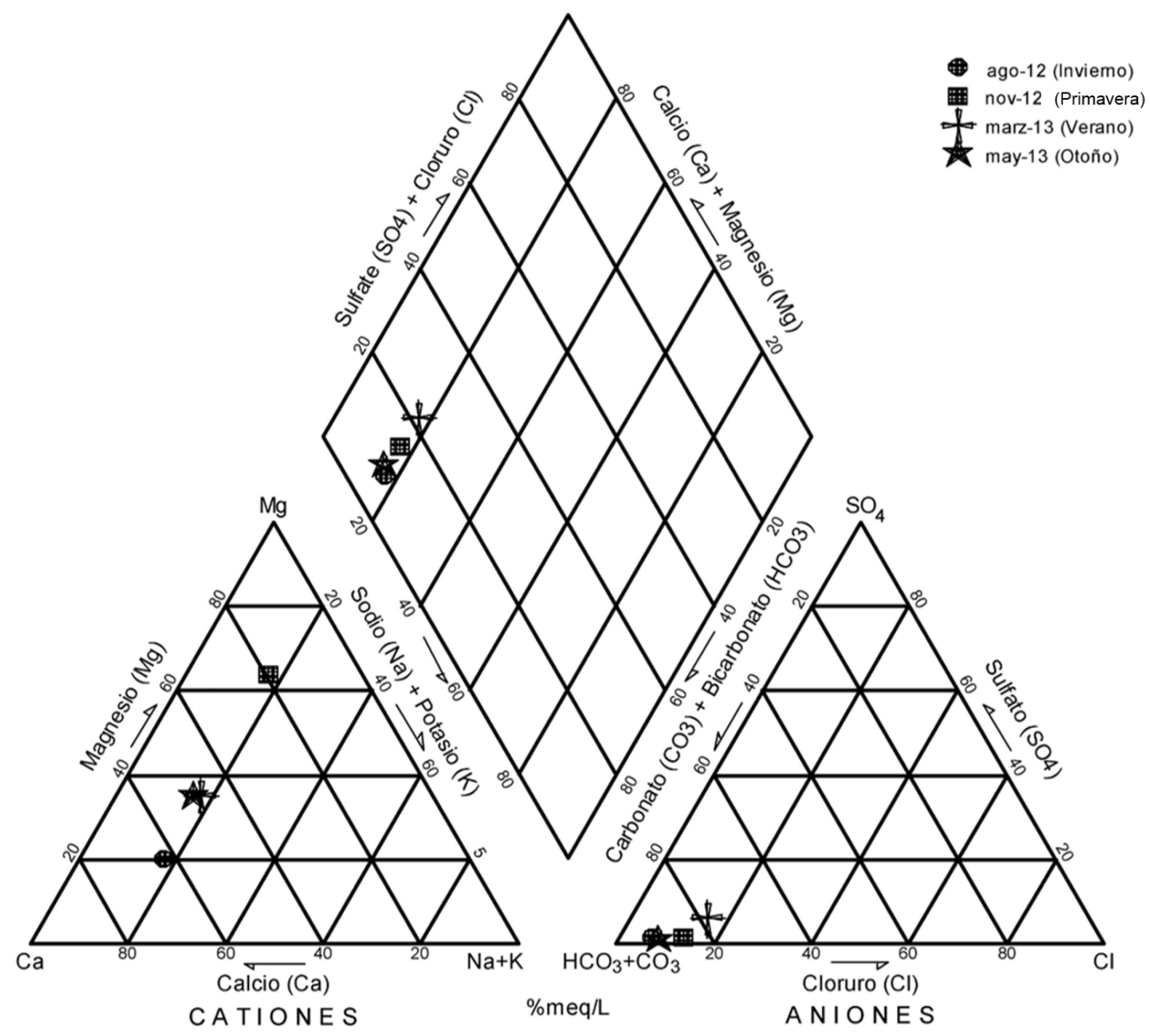

Fig. 3. Diagrama de Piper-Hill-Langelier

Fig. 3. Diagram of Piper-Hill-Langelier

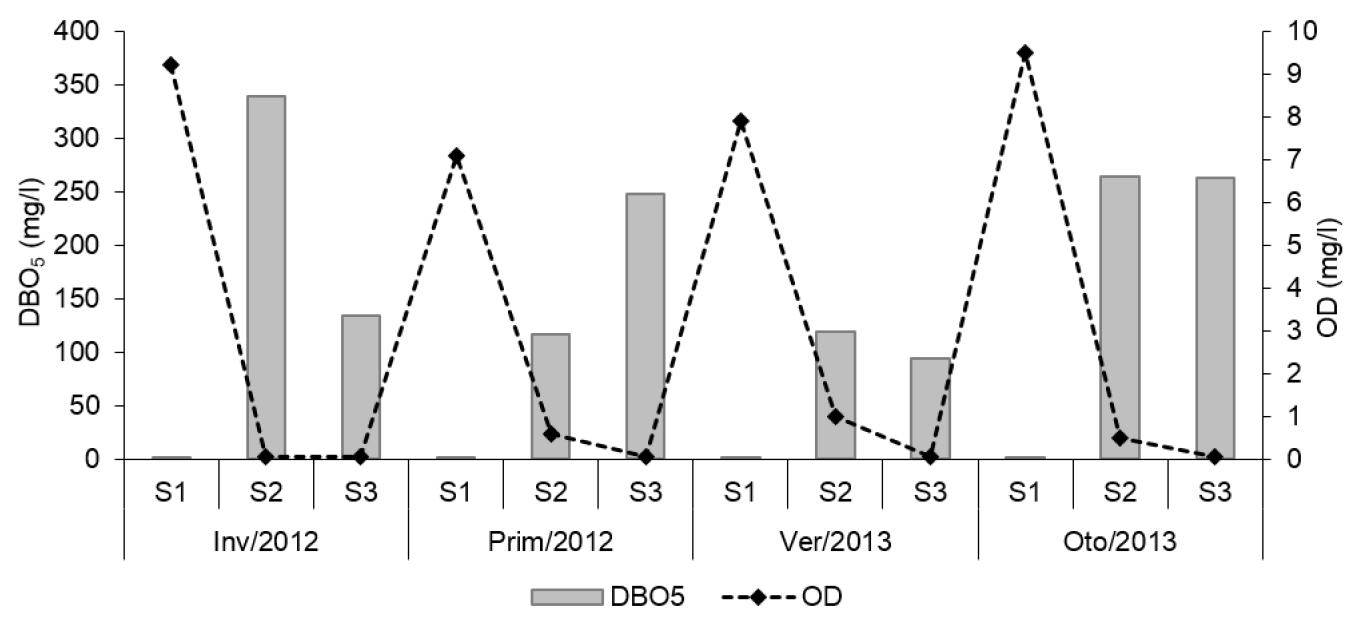

Fig. 4. Fluctuaciones de la concentración de oxígeno disuelto y demanda bioquímica de oxígeno del arroyo Calimayo Fig. 4. Fluctuations in dissolved oxygen concentration and biochemical oxygen demand of the Calimayo stream. 
Tabla 3. Riqueza fitoplanctónica y codificación de especies utilizadas en RDA. * taxones registrados en el 100\% de las muestras. - Especies poco frecuentes encontradas en menos del 30\%. El resto osciló entre un 50-75\% de presencia en las muestras analizadas.

Table 3. Phytoplankton richness and coding of species used in RDA. * taxa registered in 100\% of the samples. "

Uncommon species found in less than 30\%. The rest ranged between $50-75 \%$ presence in the analyzed samples.

\begin{tabular}{|c|c|c|c|c|c|c|c|c|c|c|c|c|c|}
\hline \multirow[b]{2}{*}{ Clase Bacillariophyceae } & \multicolumn{3}{|c|}{ Inv/12 } & \multicolumn{3}{|c|}{ Prim/12 } & \multicolumn{3}{|c|}{ Ver/12 } & \multicolumn{3}{|c|}{ Oto/12 } & \multirow[t]{2}{*}{$\begin{array}{l}\text { Cod. } \\
\text { RDA }\end{array}$} \\
\hline & S1 & S2 & S3 & s1 & S2 & S3 & s1 & S2 & S3 & S1 & S2 & S3 & \\
\hline Achnanthes brevipes Agardh & $x$ & & & $x$ & & & & & & $x$ & & & Acbr \\
\hline A. inflata (Kützing) Grunow• & $x$ & & & & & & & & & $x$ & & & \\
\hline Amphipleura lindheimerii Grunow & $\mathrm{x}$ & & & $x$ & & & $x$ & & & $x$ & & & Amli \\
\hline Amphora ovalis (Kützing) Kützing & & & & $x$ & $x$ & & & & & $x$ & & & \\
\hline $\begin{array}{l}\text { Cocconeis placentula var. euglypta } \\
\text { (Ehrenberg ) Grunow }{ }^{*}\end{array}$ & $x$ & $x$ & & $x$ & $x$ & $x$ & $x$ & $x$ & & $x$ & $x$ & $x$ & Coeu \\
\hline $\begin{array}{l}\text { C. placentula var. lineata (Ehrenberg ) } \\
\text { Van Heurck }\end{array}$ & $x$ & $x$ & $x$ & $x$ & $x$ & $x$ & $x$ & $x$ & $x$ & $\mathrm{x}$ & $x$ & $x$ & Coli \\
\hline Craticula ambigua (Kützing) Mann & $\mathrm{X}$ & & & & & & & & $x$ & $x$ & & $x$ & Cram \\
\hline Cyclotella meneghiniana Kützing. & $x$ & & $\mathrm{X}$ & & $x$ & $x$ & & & & & $x$ & $x$ & \\
\hline Cymatopleura solea (Brébisson) Smith- & & $x$ & & & & & & $x$ & & & $x$ & & Cyso \\
\hline Cymbella affinis Kützing* & $\mathrm{x}$ & $\mathrm{X}$ & $\mathrm{x}$ & $x$ & $x$ & $x$ & $x$ & $x$ & $\mathrm{x}$ & $x$ & $x$ & $x$ & Cyaf \\
\hline C. cymbiformis Agardh & & $x$ & $\mathrm{X}$ & & $x$ & $x$ & & $x$ & & $x$ & $x$ & $x$ & Сусу \\
\hline Diadesmis confervaceae Kützing & $x$ & & & $x$ & & & $x$ & & & $x$ & & & \\
\hline Diatoma moniliformis Kützing & $\mathrm{X}$ & & & $x$ & & & $x$ & & & & & & \\
\hline D. vulgaris Bory & $x$ & $x$ & $x$ & $x$ & $x$ & $x$ & $x$ & $x$ & $x$ & $x$ & $x$ & $x$ & \\
\hline $\begin{array}{l}\text { Encyonema sileciacum (Bleisch) } \\
\text { D.G.Mann }\end{array}$ & $x$ & & & $x$ & & & $x$ & & & $x$ & & & \\
\hline E. vulgare (Kützing) D. G. Mann• & $\mathrm{x}$ & & & $x$ & & & & & & & & & \\
\hline Epithemia turgida (Ehrenberg) Kützing• & $x$ & & & & & & & & & $x$ & & & \\
\hline Eunotia falax Cleve. & $\mathrm{X}$ & & & $x$ & & & & & & & & & \\
\hline E. pectinalis (Kützing) Rabenhorst & $x$ & & & & & $x$ & $x$ & & $x$ & & & & \\
\hline Gomphonema affine Kützing• & $x$ & & & & & & & & & $x$ & & & \\
\hline $\begin{array}{l}\text { G. affinopsis Metzeltin \& García- } \\
\text { Rodríguez: }\end{array}$ & & & & & & & & & & $x$ & & & \\
\hline G. capitatum Ehrenberg & $\mathrm{X}$ & & & $x$ & $x$ & & & & & & & & Goca \\
\hline G. clavatum Ehrenberg & & & & $x$ & & & & & $x$ & & & & \\
\hline G. gracile Ehrenberg. & $x$ & & & & & & $x$ & & & & & & \\
\hline G. parvulum (Kützing) Kützing & $x$ & $x$ & & $x$ & $x$ & $x$ & $x$ & $x$ & $x$ & $x$ & $x$ & $x$ & Gopa \\
\hline G. pseudoaugur Lange-Bertalot & $\mathrm{X}$ & & & $x$ & & $x$ & & $x$ & $\mathrm{X}$ & & & $\mathrm{X}$ & \\
\hline G. subclavatum (Grunow) Grunow & & $\mathrm{X}$ & & $x$ & $x$ & & & & & & & & \\
\hline G. truncatum Ehrenberg & $x$ & & & $x$ & & $x$ & & & & & & & Gotr \\
\hline Halamphora veneta (Kützing) Levkov & $x$ & & & $x$ & & & & & & $x$ & & $x$ & \\
\hline $\begin{array}{l}\text { Hantzschia amphioxis (Ehrenberg) } \\
\text { Grunow }\end{array}$ & $x$ & & & $x$ & & & $\mathrm{x}$ & & & & & & \\
\hline
\end{tabular}




\begin{tabular}{|c|c|c|c|c|c|c|c|c|c|c|c|c|}
\hline \multirow[b]{2}{*}{ Clase Bacillariophyceae } & \multicolumn{2}{|c|}{ Inv/12 } & \multicolumn{3}{|c|}{ Prim/12 } & \multicolumn{3}{|c|}{ Ver/12 } & \multicolumn{3}{|c|}{ Oto/12 } & \multirow[t]{2}{*}{$\begin{array}{l}\text { Cod. } \\
\text { RDA }\end{array}$} \\
\hline & S1 & S2 S3 & s1 & S2 & S3 & s1 & S2 & S3 & s1 & S2 & S3 & \\
\hline $\begin{array}{l}\text { Hippodonta capitata subsp. } \\
\text { iberoamericana Metzeltin, Lange- } \\
\text { Bertalot \& García Rodriguez. }\end{array}$ & $\mathrm{x}$ & & & & & $x$ & & & $x$ & & & \\
\hline $\begin{array}{l}\text { Lemnicola hungarica (Grunow) Round } \\
\text { \& Basson: }\end{array}$ & & & $\mathrm{x}$ & & & $x$ & & & & & & \\
\hline Luticola mutica (Kützing) D. G. Mann & $x$ & & $x$ & & & $x$ & & & $x$ & & & Lumu \\
\hline Melosira varians Agardh & $x$ & & $X$ & $x$ & & $x$ & & & $x$ & & & Meva \\
\hline Navicula cryptocephala Kützing & & $x$ & & $x$ & $x$ & & $x$ & $x$ & & $x$ & $x$ & \\
\hline N. gregaria Donkin & $\mathrm{X}$ & & $\mathrm{X}$ & & & & & & $\mathrm{X}$ & & & \\
\hline N. peregrina (Ehrenberg) Kützing• & $x$ & & & & & & & & $x$ & & & Nape \\
\hline N. radiosa Kützing. & $x$ & & & & & & & & $x$ & & & \\
\hline N. tripunctata (O. F. Müller) Bory & $x$ & & $X$ & & & $x$ & & & $x$ & & & \\
\hline N. trivialis Lange-Bertalot: & $x$ & & $X$ & & & & & & & & & Natr \\
\hline N. veneta Kützing & $x$ & & $x$ & & & & & & & & & Nave \\
\hline Navicula sp. & $x$ & & & & $x$ & & & & & & & Nasp \\
\hline Nitzschia acicularis (Kützing) Smith- & & & & & & & & & $x$ & & & \\
\hline N. amphibia Grunow & $x$ & & $X$ & & & & & & $x$ & & & Niam \\
\hline N. brevissima Grunow & $x$ & & $X$ & & & $x$ & & & & & & \\
\hline N. capitellata Hustedt. & $x$ & & & & & & & & $x$ & & & \\
\hline $\begin{array}{l}\text { N. intermedia Hantzsch ex Cleve \& } \\
\text { Grunow }\end{array}$ & $x$ & & & $x$ & $x$ & $x$ & & $x$ & & & & Niim \\
\hline N. linearis (Agardh) Smith & $x$ & & $X$ & & & $x$ & & $x$ & $x$ & & $x$ & Nili \\
\hline N. palea (Kützing) Smith & $x$ & $x$ & $x$ & $x$ & $x$ & $x$ & $x$ & $x$ & $x$ & & $x$ & Nipa \\
\hline N. sigmoidea (Nitzsch) Smith & & & & & $x$ & & & & & $x$ & & \\
\hline $\begin{array}{l}\text { Orthoseira roseanna (Rabenhorst) } \\
\text { O'Meara }\end{array}$ & $X$ & & $X$ & & & $x$ & & & $x$ & & & \\
\hline $\begin{array}{l}\text { Pinnularia divergentissima (Grunow) } \\
\text { Cleve- }\end{array}$ & & & & & $x$ & & & & & & & \\
\hline P. viridis (Nitzsch) Ehrenberg & $x$ & & & & $x$ & & & & $x$ & & $x$ & Pivi \\
\hline Placoneis elginensis (Gregory) Cox & $x$ & & $X$ & & & & & & $x$ & & & Plel \\
\hline $\begin{array}{l}\text { Reimeria uniseriata Sala, Guerrero \& } \\
\text { Ferrario }\end{array}$ & $x$ & & $X$ & & & $x$ & & & $x$ & $x$ & & Reun \\
\hline $\begin{array}{l}\text { Rhoicosphenia abbreviata (Agardh) } \\
\text { Lange-Bertalot }\end{array}$ & $x$ & $x$ & $x$ & $x$ & & $x$ & & $x$ & $x$ & & & Rhab \\
\hline Sellaphora pupula Kützing & $x$ & & $\mathrm{X}$ & & & & & $x$ & $x$ & & & Sepu \\
\hline Surirella ovalis Brébisson & $\mathrm{X}$ & & $\mathrm{X}$ & $\mathrm{X}$ & $x$ & $x$ & & & $x$ & & & Suov \\
\hline Ulnaria ulna (Nitzsch) Compère & $x$ & & $\mathrm{X}$ & $x$ & & $x$ & $x$ & & $x$ & $x$ & $x$ & Ulul \\
\hline \multicolumn{13}{|l|}{ Clase Chlorophyceae } \\
\hline $\begin{array}{l}\text { Cladophora glomerata (Linnaeus) } \\
\text { Kützing }\end{array}$ & $x$ & & & & & $x$ & & & $x$ & & & \\
\hline Closterium gracile Brébisson ex Ralfs & & & & & & & $x$ & & $x$ & $x$ & & Clgr \\
\hline
\end{tabular}




\begin{tabular}{|c|c|c|c|c|c|c|c|c|c|c|c|c|}
\hline & \multicolumn{2}{|c|}{ Inv/12 } & \multicolumn{3}{|c|}{ Prim/12 } & \multicolumn{3}{|c|}{ Ver/12 } & \multicolumn{3}{|c|}{ Oto/12 } & \multirow[t]{2}{*}{$\begin{array}{l}\text { Cod. } \\
\text { RDA }\end{array}$} \\
\hline & S1 & S2 S3 & s1 & S2 & S3 & s1 & $\mathbf{S 2}$ & S3 & s1 & S2 & S3 & \\
\hline C. moniliferum (Bory) Ehrenberg. & $x$ & & & & & & & & $x$ & & & \\
\hline Oedogonium sp. & $x$ & & $\mathrm{X}$ & $\mathrm{X}$ & & & & & $x$ & & & Oesp \\
\hline Pyrobotrys sp. & & $x$ & & $\mathrm{X}$ & $\mathrm{X}$ & & $x$ & $x$ & $x$ & $\mathrm{X}$ & $x$ & Pysp \\
\hline $\begin{array}{l}\text { Scenedesmus quadricauda (Turpin) } \\
\text { Brébisson }\end{array}$ & & $\mathrm{x}$ & & $\mathrm{X}$ & $\mathrm{X}$ & & $\mathrm{x}$ & $x$ & & $\mathrm{X}$ & $\mathrm{X}$ & Scqu \\
\hline S. falcatus Chodat & & $x$ & & $\mathrm{X}$ & & & & $x$ & & $\mathrm{X}$ & & Scfa \\
\hline Sphaerocystis sp. & & & & $\mathrm{X}$ & & & $\mathrm{X}$ & & & $\mathrm{X}$ & & Spsp \\
\hline Stigeoclonium sp. & $\mathrm{X}$ & & & $\mathrm{X}$ & & & & & & & & \\
\hline \multicolumn{13}{|l|}{ Clase Euglenophyceae } \\
\hline Euglena sp. & & $x$ & & $\mathrm{X}$ & $\mathrm{X}$ & & $\mathrm{x}$ & & & $\mathrm{X}$ & $x$ & Eusp \\
\hline Phacus longicauda Dujardin & & & & $\mathrm{X}$ & & & & & & & & Phlo \\
\hline P. orbicularis Hübner. & & $x$ & & $\mathrm{X}$ & & & $x$ & & & $\mathrm{X}$ & & Phor \\
\hline Trachelomonas oblonga Lemmermann & & $\mathrm{x}$ & & $\mathrm{X}$ & $\mathrm{X}$ & & $\mathrm{X}$ & $x$ & & & $\mathrm{X}$ & Trob \\
\hline \multicolumn{13}{|l|}{ Clase Cyanophyceae } \\
\hline Lyngbya birgei Smith* & $\mathrm{x}$ & $\mathrm{x}$ & $\mathrm{x}$ & $\mathrm{X}$ & $\mathrm{X}$ & $\mathrm{x}$ & $\mathrm{x}$ & $x$ & $x$ & $\mathrm{X}$ & $\mathrm{X}$ & Lybi \\
\hline L. limnetica Lemmermann & $\mathrm{X}$ & $x$ & $\mathrm{X}$ & $X$ & $\mathrm{X}$ & $x$ & $\mathrm{X}$ & $x$ & $x$ & $\mathrm{X}$ & $x$ & Lyli \\
\hline $\begin{array}{l}\text { Merismopedia glauca (Ehrenberg) } \\
\text { Kützing }\end{array}$ & $x$ & & $X$ & & & & & & $x$ & & & \\
\hline Oscillatoria acuminata Gomont & $\mathrm{X}$ & & & $\mathrm{X}$ & & & $\mathrm{X}$ & $x$ & & $\mathrm{X}$ & & Osac \\
\hline O. limosa C. Agardh ex Gomont & & $x$ & & & $\mathrm{X}$ & $x$ & & & $x$ & $X$ & $x$ & Osli \\
\hline O. tenuis C. Agardh ex Gomont* & $x$ & $x$ & $x$ & $\mathrm{X}$ & $\mathrm{X}$ & $x$ & $x$ & $x$ & $x$ & $\mathrm{X}$ & $x$ & Oste \\
\hline Phormidium ambiguum Gomont & $\mathrm{X}$ & & $\mathrm{X}$ & & $\mathrm{X}$ & $\mathrm{X}$ & & & & $\mathrm{X}$ & $\mathrm{X}$ & Pham \\
\hline
\end{tabular}

varianza (inercia $=0,44 ; \mathrm{p}<0,05)$. E1 33\% de la variación se refleja en el primer eje canónico (autovalor $=0,15$ ) el cual separa dos grupos de taxones uno formado por Melosira varians (Meva), Navicula tripunctata (Natp), Reimeria uniseriata (Reun), Rhoicosphenia abbreviata (Rhab) y Ulnaria ulna (Ulul), (grupo 1, especies sensibles, eje RDA1 negativo) y otro grupo formado por Cymbella cymbiformis (Cycy), Lyngbya birgei (Lybi), Lyngbya limnetica (Lyli), Navicula sp. 1 (Nasp), Nitzschia palea (Nipal), Oscillatoria limosa (Osli), Oscillatoria tenuis (Oste) y Pyrobotrys sp. (Pysp) (grupo 2, taxones tolerantes, eje RDA1 positivo). La Figura 8 muestra que las abundancias de las especies del grupo 1 se asocian al sitio 1 independientemente de la fecha de muestreo, con mayores valores de $\mathrm{pH}, \mathrm{OD}, \mathrm{CO}_{3}{ }^{2-} \mathrm{y}$ menores valores de temperatura, cloruro $\left(\mathrm{Cl}^{-}\right), \mathrm{DBO}_{5}, \mathrm{HCO}_{3}^{-}$, $\mathrm{SO}_{4}^{2-}$ y K ${ }^{+}$, mientras que el grupo 2 se registró en los sitios 2 y 3 , afectados por efluentes, en condiciones ambientales inversas a las del grupo 1. El eje RDA2 representa un $20 \%$ de la varianza explicada (autovalor $=0,09$ ) pero los valores de correlación son bajos comparados con el eje RDA1 (Tabla 5).

Se detectó solamente una asociación lineal positiva entre la abundancia relativa del grupo 1 (especies sensibles) y la biomasa ( $\mathrm{r}=0,66$; $\mathrm{p}<0,05)$. Se destaca también una correlación negativa entre abundancia relativa del grupo 1 y grupo $2(r=-0,86 ; p<0,05)$.

Las curvas de abundancia-dominancia para cada sitio difirieron entre sí en cuanto a la composición y la dominancia específica. La 


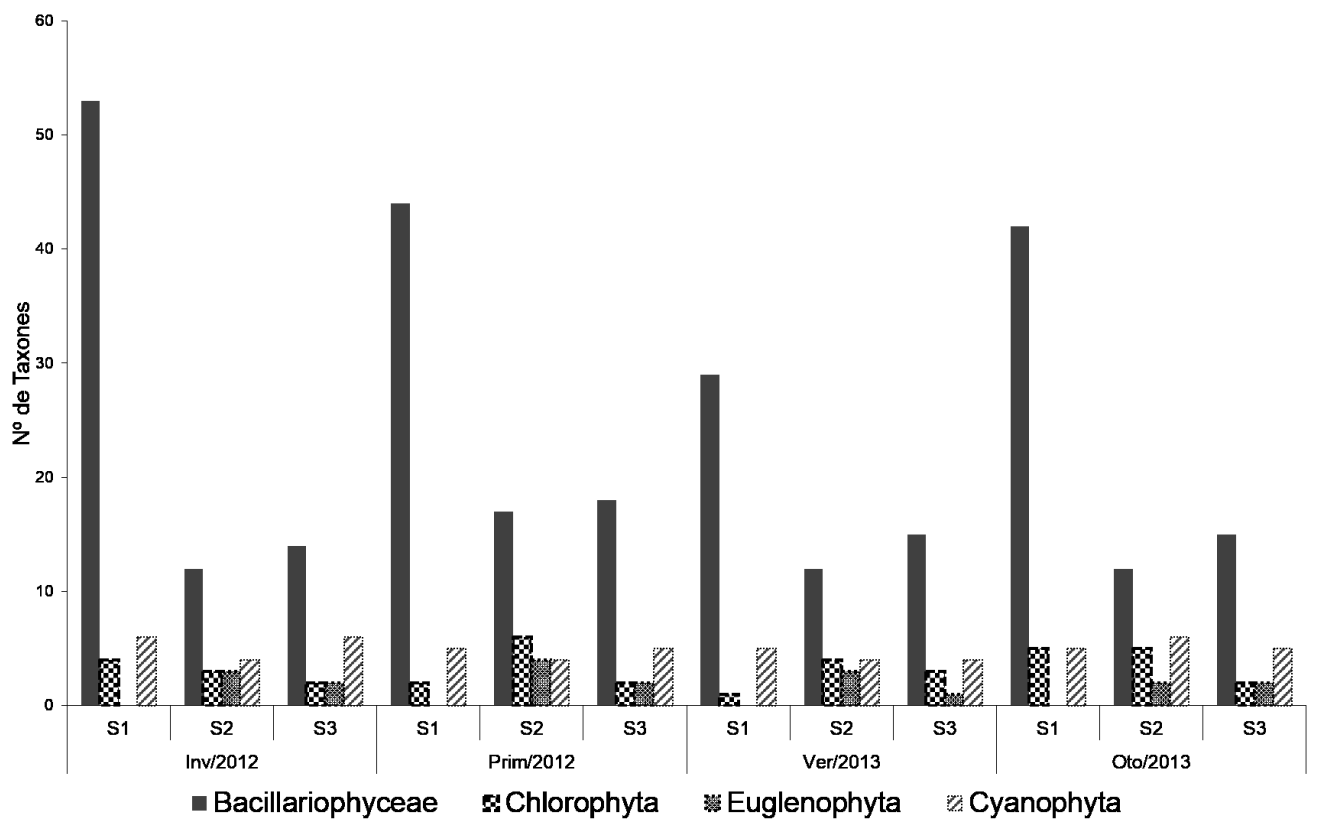

Fig. 5. Riqueza fitoplanctónica de los sitios estudiados.

Fig. 5. Phytoplanktonic richness of the sites studied.

Tabla 4. Valores de los distintos índices bióticos registrados en el arroyo Calimayo.

Table 4. Values of the different biotic indices registered in the Calimayo stream.

\begin{tabular}{|lcccccccccccc|}
\hline & & Inv/12 & \multicolumn{3}{c}{ Prim/12 } & \multicolumn{3}{c|}{ Ver/13 } & \multicolumn{3}{c|}{ Oto/13 } \\
& $\mathbf{S 1}$ & $\mathbf{S 2}$ & $\mathbf{S 3}$ & $\mathbf{S 1}$ & $\mathbf{S 2}$ & $\mathbf{S 3}$ & $\mathbf{S 1}$ & $\mathbf{S 2}$ & $\mathbf{S 3}$ & $\mathbf{S 1}$ & $\mathbf{S 2}$ & $\mathbf{S 3}$ \\
I. Diversidad & 3,2 & 1,2 & 2,2 & 3,8 & 3,3 & 3,1 & 2,4 & 2,8 & 2,3 & 2,8 & 2,6 & 2,1 \\
I. Equidad & 0,55 & 1 & 0,59 & 0,44 & 0,45 & 0,46 & 0,64 & 0,48 & 0,58 & 0,61 & 0,51 & 0,65 \\
ICOMO & 0,03 & 1 & 1 & 0,1 & 1 & 1 & 0,2 & 1 & 1 & 0,1 & 1 & 1 \\
I. Sap. & 1,8 & 3,4 & 3,3 & 1,7 & 3,2 & 3,2 & 1,7 & 3,1 & 3,2 & 1,8 & 3 & 3,3 \\
\hline
\end{tabular}

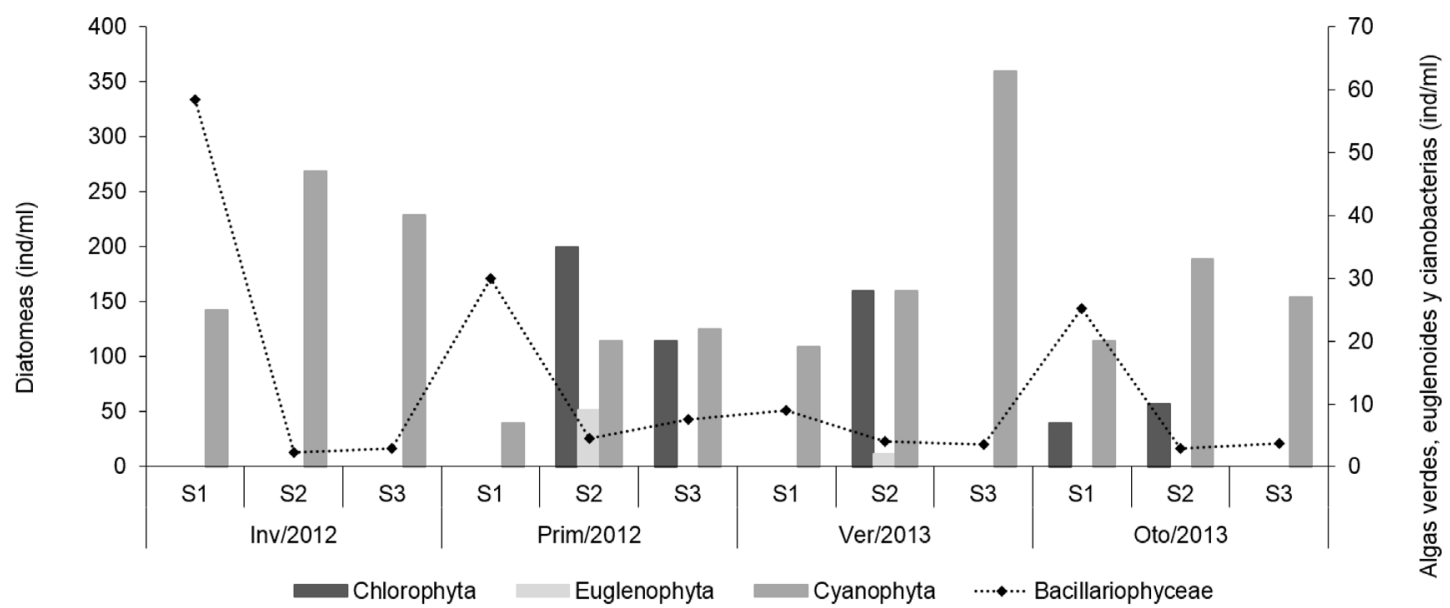

Fig. 6. Fluctuaciones de la abundancia algal durante el periodo estudiado.

Fig. 6. Fluctuations in algal abundance during the period studied. 


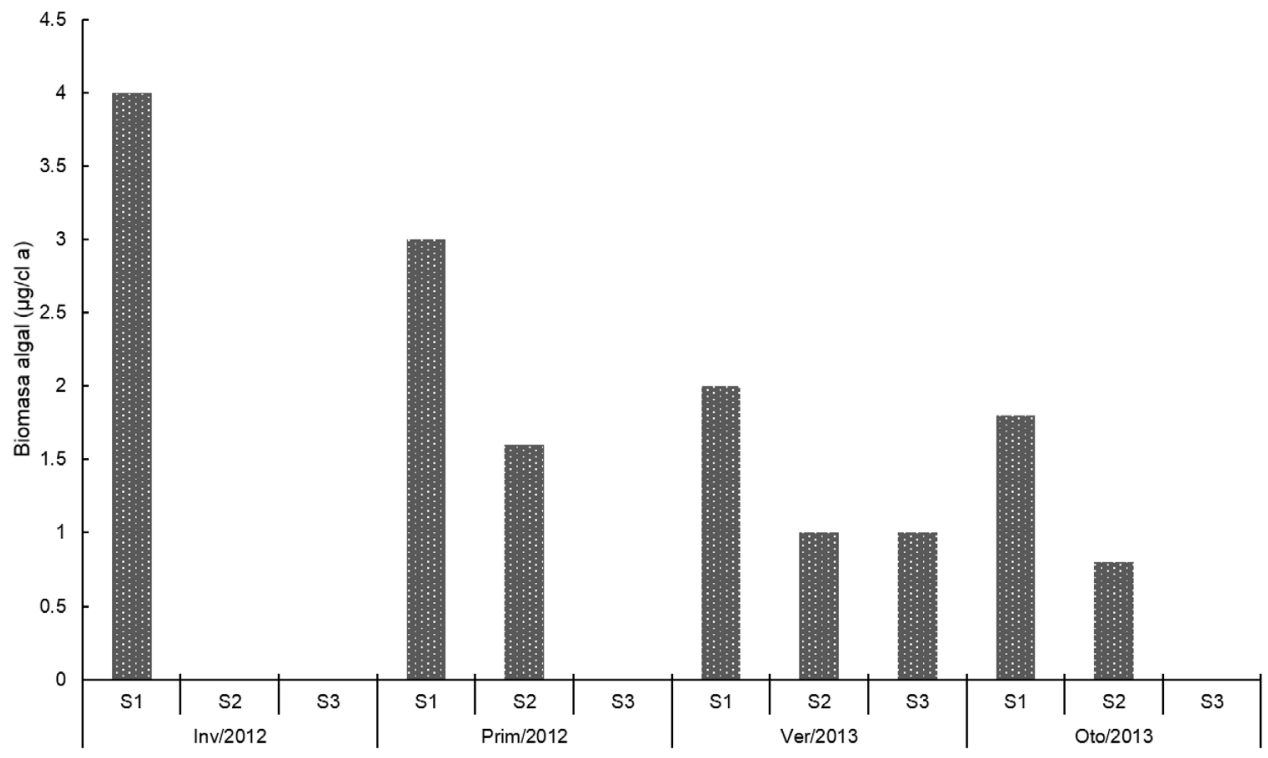

Fig. 7. Variación de la biomasa algal.

Fig. 7. Variation of algal biomass.

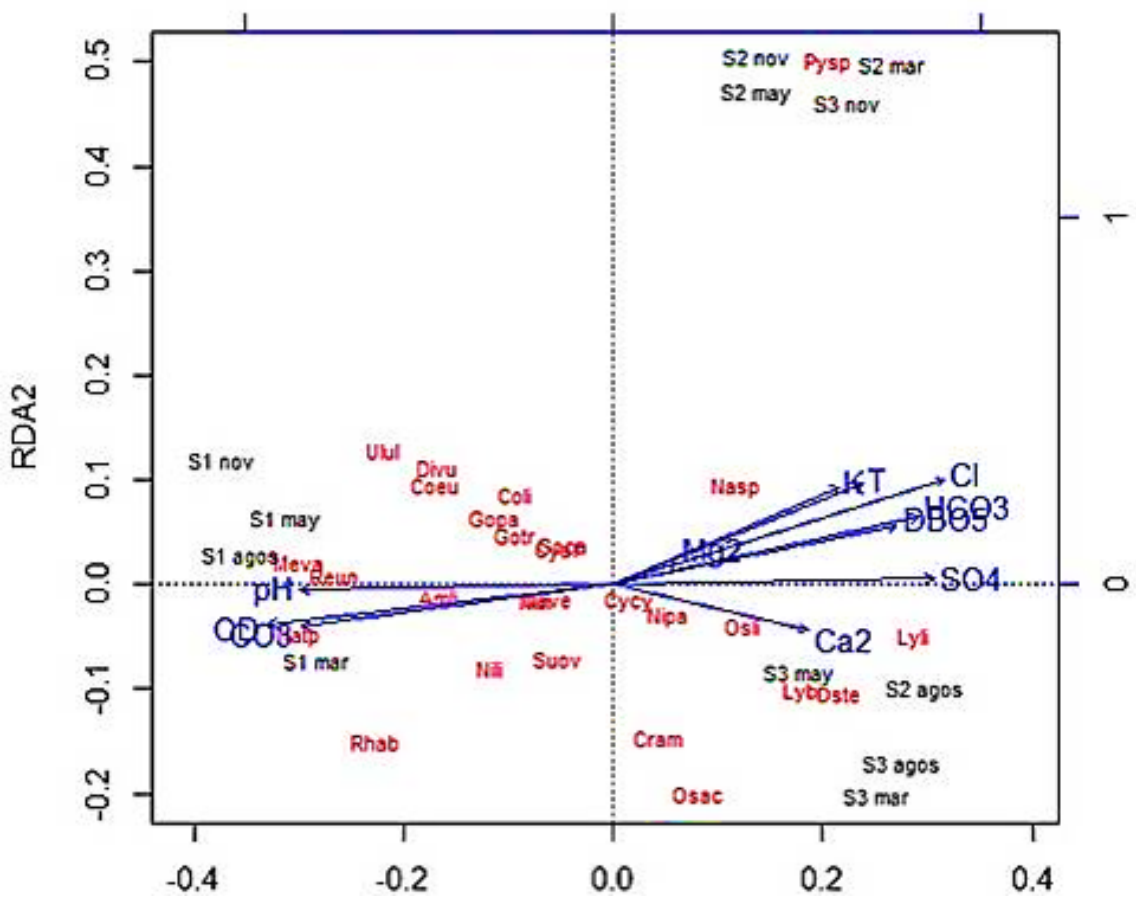

Fig. 8. Triplot de RDA: sitio 1 (antes de descarga), sitios 2 y 3 (después de descarga) durante cuatro estaciones: agos (invierno/2012), nov (primavera /2012), mar (verano/2013) y may (otoño/2013). Referencias de los vectores: $\mathrm{K}$ (potasio), $\mathrm{T}$ (temperatura), $\mathrm{Cl}^{-}$(cloruro), $\mathrm{HCO}_{3}^{-}$(Bicarbonato), $\mathrm{DBO}_{5}$ (demanda bioquímica de oxígeno), $\mathrm{Mg}^{2+}$ (Magnesio), $\mathrm{SO}^{2+}{ }_{4}$ (Sulfato), $\mathrm{Ca}^{2+}$ (Calcio), $\mathrm{pH}, \mathrm{OD}$ (Oxígeno disuelto), $\mathrm{CO}_{3}^{-}$(Carbonato). Ver codificación de especies en Tabla 3.

Fig. 8. Triplot of RDA: site 1 (before download), sites 2 and 3 (after download) during four seasons: August (winter/2012), nov (spring/2012), march (summer/2013) and may (autumn/2013). Vectors references: K (potassium), T (temperature), $\mathrm{Cl}^{-}$(chloride), $\mathrm{HCO}_{3}^{-}$(Bicarbonate), $\mathrm{DBO}_{5}$ (Biochemical Oxygen Demand), $\mathrm{Mg}^{2+}$ (Magnesium), $\mathrm{SO}^{2+}{ }_{4}$ (Sulfate), $\mathrm{Ca}^{2+}$ (Calcium), pH , OD (dissolved oxygen), $\mathrm{CO}_{3}^{-}$(Carbonate). See Coding of species in Table 3. 
Figura 9 muestra una mayor equitatividad en la distribución de las abundancias de las especies en el sitio 1 (curva menos vertical) que en los sitios 2 y 3 más perturbados. Además, se destaca que las cuatro especies más abundantes en el sitio 1: Melosira varians (Meva), Ulnaria ulna (Ulul), Navicula tripunctata (Natp) y Reimeria uniseriata (Reun) no coinciden con las cuatro especies más abundantes en los sitios 2 y 3 que correspondieron a Pyrobotrys sp. (Pysp), Oscillatoria tenuis (Oste), Lyngbya limnetica (Lyli) y Lyngbya birgei (Lybi).

\section{Discusión y Conclusiones}

La geoquímica y biota de ríos y arroyos reflejan lo que sucede en el conjunto del territorio, en tal sentido pueden aportar información del estado de conservación y "salud" de la cuenca (Margalef, 1997). La gran variabilidad natural, espacial y temporal en los ecosistemas fluviales hace que la evaluación de la calidad del agua y los impactos humanos resulte compleja (Sosa et al., 2011).

Los datos de temperatura en S1 fueron coincidentes con la estacionalidad anual y se corresponden con la radiación solar propia de cada época, semejante a lo encontrado por Isasmendi (2016) para los ríos Blanquito, El Mollar y La Ovejería en Tucumán; mientras que en los sitios 2 y 3 los registros térmicos se vieron incrementados por la descarga poluta, situación concordante con los arroyos El Gato y Rodríguez en Buenos Aires, sometidos al vertido de aguas residuales de diversas fábricas (Mercado, 2001).

Las aguas del arroyo se mantuvieron alcalinas durante todo el periodo analizado, sin embargo, la tendencia decreciente de los valores aguas abajo de la papelera sea, probablemente, el resultado de la descomposición de la materia orgánica y de compuestos clorados, sulfurados, etc., vertida en el arroyo tal como reportan Pizarro \& Alemanni (2005) y Plataroti (2010), para el río Luján sometido a contaminación por un parque industrial.

La conductividad eléctrica de la mayoría de las aguas continentales varía entre 10$1.000 \mu \mathrm{S} \mathrm{cm}^{-1}$, pero puede exceder este valor especialmente si son aguas polutas o porque reciben grandes cantidades de sedimento por escurrimiento (Chapman \& Kimstach, 1992). La CE fue muy elevada en los sitios ubicados después del vertido. Los valores

Tabla 5. Coeficientes de correlación intra conjunto de los ejes canónicos y variables ambientales Table 5. Coefficients of intra-set correlation of canonical axes and environmental variables.

\begin{tabular}{|lcc|}
\hline \multicolumn{1}{|c}{$\begin{array}{c}\text { Código de las variables } \\
\text { ambientales }\end{array}$} & Coeficiente de correlación del set de datos \\
Temperatura (T) & 0,68 & RDA2 \\
$\mathrm{pH}$ & $-0,852$ & 0,274 \\
Oxígeno disuelto (OD) & $-0,94$ & $-0,016$ \\
$\mathrm{DBO}_{5}$ & 0,77 & $-0,107$ \\
$\mathrm{Cl}^{-}$ & 0,902 & 0,158 \\
$\mathrm{HCO}_{3}^{-}$ & 0,831 & 0,286 \\
$\mathrm{CO}_{3}{ }^{2-}$ & $-0,847$ & 0,183 \\
$\mathrm{SO}_{4}{ }^{2-}$ & 0,871 & $-0,117$ \\
$\mathrm{Ca}^{2+}$ & 0,53 & 0,016 \\
$\mathrm{~K}^{+}$ & 0,613 & $-0,124$ \\
$\mathrm{Mg}^{2+}$ & 0,174 & 0,264 \\
$\mathrm{NO}_{3}^{-}$ & 0,11 & 0,057 \\
\hline
\end{tabular}




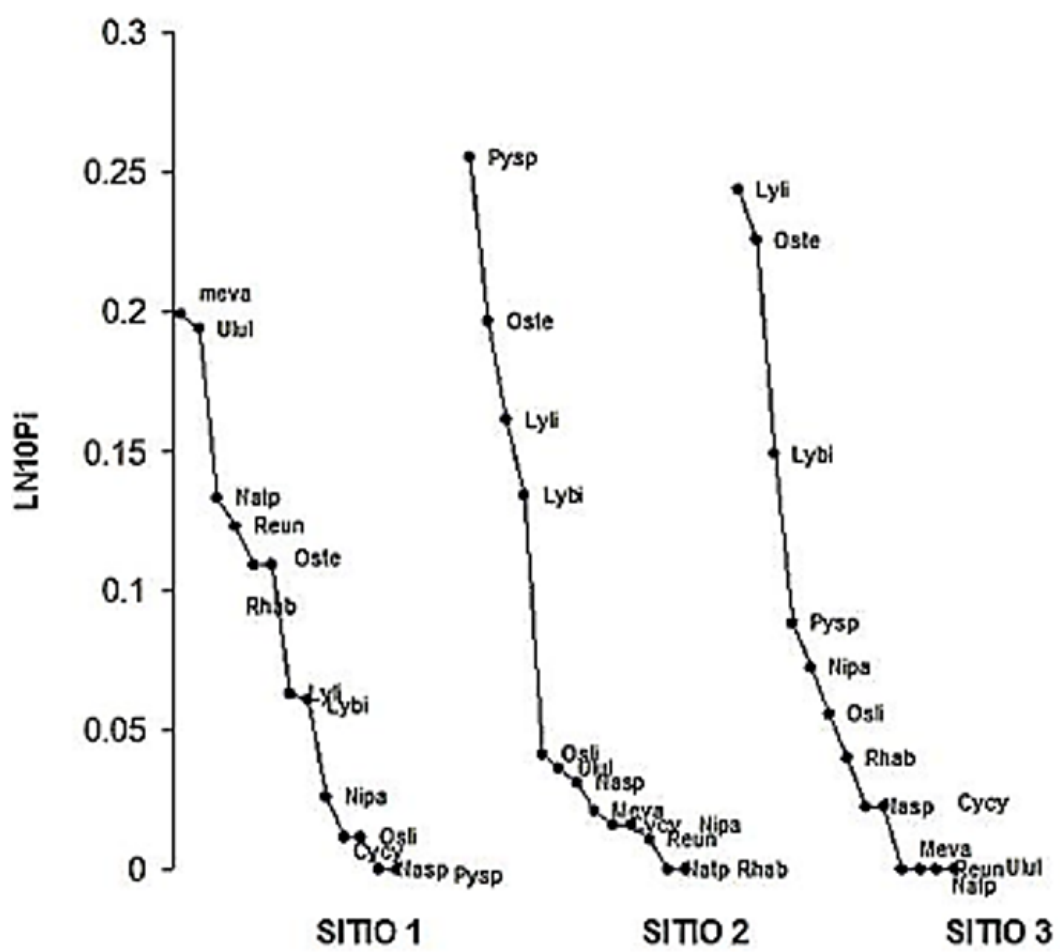

Fig. 9. Curva de abundancia-dominancia de especies para cada sitio del arroyo Calimayo. Ver codificación de especies en Tabla 3.

Fig. 9. Abundance-species dominance curve for each site of the Calimayo stream. See Coding of species in Table 3.

en S1 se encontraron en el rango de una mineralización media (hasta $666 \mu \mathrm{S} \mathrm{cm}^{-1}$ ) con algunas variaciones estacionales, mientras que para S2 y S3 los registros correspondieron a una mineralización excesiva (más de $1.000 \mu \mathrm{S}$ $\mathrm{cm}^{-1}$ ) (Rodier, 1990). Estos datos coinciden con los encontrados por González \& Domínguez (1994), Padilla Torres et al. (1996), Isasmendi et al. (2002) y Mirande (2006) para distintos ríos de Tucumán, en los cuales la CE fue muy elevada por acción de efluentes polutos industriales. En relación al tipo de agua en S1 se asoció a la litología por la cual discurre el arroyo mientras que en los otros dos sitios (S2 y S3) se vio un incremento en la cantidad de cloruro y sodio evidenciando una modificación por el vuelco de efluentes, en concordancia a lo encontrado por Rodríguez et al. (2002) para el río Chocancharava (Córdoba), el cual recibe contaminantes de origen urbano e industrial en algunos sectores de su recorrido.

El oxígeno disuelto presentó un patrón de variación estacional en el sitio 1 ya que su concentración disminuyó con el aumento de temperatura en los meses cálidos. En S2 y S3 se registró una disminución de los niveles de oxígeno, lo que estaría asociado a la carga orgánica y el contenido de contaminantes, coincidente con lo encontrado por diversos autores entre los que podemos citar a Plataroti (2010), quien analizó la calidad del agua en varios sitios del tramo medio del río Luján.

Los altos registros de $\mathrm{DBO}_{5}$ en $\mathrm{S} 2$ y S3 se puede deber a la presencia de compuestos orgánicos de degradación lenta (por ejemplo la celulosa), como así también a compuestos inorgánicos reductores $\left(\mathrm{Fe}^{2+}\right.$ y $\left.\mathrm{Mn}^{2+}\right)$ (Peirce, 1998; Meybeck, 2003). Probablemente, el vertido de la papelera, generó un aporte de este tipo de sustancias que no pudieron ser biodegradadas totalmente. Estas mismas observaciones fueron registradas por Giorgi et al. (1999) y Plataroti (2010) en el cauce medio y superior del río Luján. En la manufactura del papel, por cada tonelada de pulpa producida se desechan entre 20 y $350 \mathrm{~m}^{3}$ de aguas residuales 
con una composición que varía dependiendo del tipo de materia prima utilizada y de los compuestos químicos adicionados durante el proceso (Karrash et al., 2006).

La riqueza del fitoplancton presentó un marcado predominio de las diatomeas, el mayor número de especies perteneció a aquellas con simetría bilateral, siendo mínimo el número de taxones con simetría radial. Estos resultados coinciden con investigaciones realizadas en ríos de Córdoba (Martínez de Fabricius, 1996; Sosa et al., 2011). En ríos de régimen torrencial, la velocidad de corriente favorece el transporte de diatomeas pennadas del fitoplancton y derivantes del bentos (Seeligmann et al., 2001).

La presencia de cianobacterias y euglenoides en los sitios 2 y 3 estaría relacionada con la abundante materia orgánica que produce bajas concentraciones de oxígeno disuelto y elevadas de $\mathrm{NH}_{4}^{+}$, producto de las descargas de la industria, coincidente con lo encontrado por Mirande (2001) en el río Gastona (Tucumán).

Al comparar la riqueza fitoplanctónica obtenida en este trabajo con los resultados encontrados por Mirande (2006) quien analizó el fitoplancton de este arroyo en los años 19941995, se observó un incremento en el número de taxones, destacándose un recambio de especies, y se pudo apreciar un aumento de especies algales resistentes a la polución, como por ejemplo Pyrobotrys sp., la cual no fue citada por Mirande (op. cit.), y en este análisis fue altamente dominante en los sitios contaminados. Podemos afirmar que las condiciones limnológicas de este arroyo desde la década del 90 hasta la actualidad, fueron degradándose por la presencia de los efluentes industriales.

El mayor número de especies identificadas en S1 indica la gran riqueza de microalgas que pueden habitar en ese tramo del arroyo que pertenece a un ambiente de montaña y menos impactado. Los taxones encontrados, en su mayoría cosmopolitas, permiten valorar el buen estado de ese sector, recalcando la importancia de este sistema como refugio para la diversidad.

Los menores valores del índice de diversidad que se determinaron en S2 y S3 se debería principalmente a que dichos efluentes tienen efectos de toxicidad, el aumento de la conductividad eléctrica contribuiría a la especiación de metales, además que el vertido produce un incremento de nutrientes, lo que concuerdan con lo citado por Salusso \& Moraña (2002) para los ríos de la alta cuenca del Juramento en el noroeste de Argentina, afectada por efluentes polutos de origen industrial. El sitio 1 presentó una mayor diversidad de taxones, lo que podría asociarse a que en ese tramo hubo una menor concentración de sólidos en suspensión y por lo tanto una mayor transparencia lo que favoreció el desarrollo de diversas especies, situación semejante a lo citado por Isasmendi (2016) en sistemas lóticos del valle de Tafí.

El índice ICOMO reflejó que el sitio 1 de este sistema presentó una buena calidad de agua. Los valores de los sitios 2 y 3 denotaron una mala calidad debido a las altas concentraciones de materia orgánica, lo que es coincidente con lo citado por Puerto Rodríguez \& Pimentel Suárez (2006) y Torres Solano (2008) para los ríos Sampaz, Magdalena y Sancotea en Colombia. En el sitio 1 se observó la taxocenosis con mayor equitatividad (cuyos valores varían entre 0 y 1 ) en relación a los sitios contaminados donde se vio que la distribución de las abundancias entre las diferentes especies fue más heterogénea (valores $\geq 0$ ), destacando la presencia de especies dominantes tales como Nitzschia palea, Pyrobotrys sp., entre otras. Registros similares fueron citados para los ríos Arias-Arenales y Rosario (Salta) y en el río Gastona (Tucumán), sistemas lóticos sometidos a diferentes contaminantes (Mirande, 2001; Salusso \& Moraña, 2002).

El índice sapróbico calculado para el fitoplancton en S1 caracterizó al agua como oligo-ß-mesosapróbica, indicando un buen estado, estos datos coinciden con las características del tipo de agua mencionadas para ríos de Córdoba (Martínez de Fabricius et al., 2010). Los sitios 2 y 3 fueron calificados como $\alpha$-polisapróbicos, revelando una muy mala calidad del agua, con polución y eutrofización fuerte y con marcada presencia de productos de desecho debido a la actividad industrial. En estos sectores se observó un alto grado de disturbio lo que se asemeja a diversos sistemas lóticos del país que se encuentran bajo la influencia de factores contaminantes por ejemplo los ríos Luján, Reconquista, de la Plata en Buenos Aires, entre otros (O'Farrell et al., 2002, Pizarro \& Alemanni, 2005). 
La densidad algal fue máxima en invierno, tal vez debido a una disminución de las precipitaciones, coincidentes con lo encontrado por Taboada et al. (2016) para el epiliton del arroyo Mista, por Luque y Martínez de Fabricius (2003) en el río Piedra Blanca y en el río Luján por Plataroti (2010). Según De Ruyter van Steveninck et al. (1992) la disminución del flujo de corriente favorece las condiciones para el crecimiento del fitoplancton mediante el aumento de los tiempos de residencia y la menor intensidad de turbulencia. Cabe destacar que se pudo apreciar una variación en las abundancias de los distintos grupos algales: en el sitio 1 las diatomeas permanecieron con densidades elevadas durante todo el período estudiado, en S2 y S3 las Cyanophyta se incrementaron en invierno y verano, mientras que las algas verdes sobresalieron en verano y primavera en S2, de esta manera se observó una diferenciación espacial y temporal en la ficoflora.

La concentración de clorofila $a$ reflejó en gran medida, el comportamiento de las abundancias fitoplanctónicas, los valores máximos también se registraron en el sitio 1 para invierno, coincidiendo con la mayor densidad, lo que se observó en la correlación obtenida entre el grupo 1 de especies y la biomasa. Datos que coinciden con los obtenidos para el fitoplancton del arroyo Mista, en la provincia de Tucumán (Taboada et al., 2016). Las menores biomasas registradas en S2 y S3 se debería a la escasa penetración de la luz por el alto contenido de sólidos en suspensión y al efecto del vertido de iones (ej. cloruro), similar a lo encontrado por O'Farrell et al. (2002) y Pizarro \& Alemanni (2005) en ríos de la provincia de Buenos Aires, quienes concluyen que esta situación sería la limitación en el crecimiento del fitoplancton en este tipo de ambiente.

El análisis de RDA permitió diferenciar dos grupos de taxones antagónicos basándose en sus distintas respuestas a factores fisicoquímicos. El grupo 1 correspondientes a especies del S1, resultaron indicadoras de una buena calidad del agua desarrollándose en condiciones oxigénicas, baja conductividad, menor concentración de cloruro y mínima demanda bioquímica de oxígeno. Los taxones del grupo 2 (S2 y S3) resultaron tolerantes en condiciones opuestas a las del sitio 1.
Las elevadas abundancias de ciertas especies del grupo 2 en S2 y S3, tales como Nitzschia palea, Cymbella cymbiformis, Lyngbya birgei y Pyrobotrys sp., coincide con lo encontrado para los arroyos Rodríguez y El Gato en Buenos Aires (Mercado, 2001), para el río Chucul en la provincia de Córdoba (Sosa et al., 2011) y en el río Ter de España (Sabater \& Sabater, 1987), zonas con una gran carga orgánica, altos registros de conductividad debido al aporte de efluentes polutos.

Al analizar los resultados del sitio 1, se pudo apreciar que estuvo influenciado por patrones como la estacionalidad, relieve, litología y fisicoquímica del agua, denotando un buen estado ecológico durante todo el periodo analizado. La variación en la composición de la riqueza fitoplanctónica estaría relacionada con cambios estacionales. Esto mismo fue encontrado para diversos sistemas lóticos entre ellos podemos mencionar al río San Joaquín (California) donde los factores que varían con la estacionalidad contribuyen significativamente a la selección de especies, debido a su influencia sobre las tasas de crecimiento algal (Leland, 2001).

La sustitución de especies sensibles por tolerantes desde S1 a S2 y S3 se corresponde principalmente al efecto de poluentes; destacándose la dominancia de especies particulares para los distintos sitios. Esto demuestra que los cambios observados en la estructura de la taxocenosis fitoplanctónica responden a las fuerzas de perturbación y de esta manera, la composición y abundancia algal, constituyen una herramienta importante para el análisis de las condiciones ambientales en este sistema.

La evaluación biótica permitió definir la importancia de las especies fitoplanctónicas en relación con su respuesta en las diferentes condiciones ambientales del arroyo y, por tanto, este trabajo podría ser un referente de comparación regional.

Se logró diferenciar el sitio de referencia de los sitios perturbados. Los resultados mostraron que las variables bióticas y abióticas están relacionadas entre sí, por lo que la restauración de la condición ecológica de este arroyo requiere de un enfoque integral para el manejo de esta subcuenca. 


\section{Bibliografía}

ANAGNOSTIDIS, K. \& J. KOMÁREK. 1988. Modern approach to the classification system of cyanophytes. 3Oscillatoriales. Archiv für Hydrobiologie, Algological Studies/Archiv für Hydrobiologie, Supplement Volumes No. 50-53: 327 - 472.

ANTOLÍN, G. \& D. OLIVA. 2003. Caracterización del bagazo de caña de azúcar mediante análisis térmico. Inf. Tec. 14: 3-25.

APHA, AWWA \& WEF. 2005. Standard Methods for the examination of water and wastewater. American Public Health Association, 21st. ed. Washington, D. C., EUA.

ARAGÓN, M. R . \& J. M. MORALES. 2003. Species composition and invasion in NW Argentinian secondary forests: Effects of land use history, environment and landscape. J. Veg. Sc. 14: 195-204. https://doi.org/10.1111/j.1654-1103.2003.tb02144.x.

BATTARBEE, E. 1986. Diatom Analysis. Pp. 527-570. En BERGLUN, B. E. (ed.), Handbook of Holocen Palaeoecology and Palaeohydrology, pp. 527-570. J. Wiley y Sons Ltd., EUA.

BATTIN, T. J., L. A., KAPLAN, J. D. NEWBOLD \& C. M. HANSEN E. 2003. Contributions of microbial biofilms to ecosystem processes in stream mesocosmos. Nature 426: 439-442. https://doi.org/10.1038/nature02152.

BOJORGE GARCÍA M. G. \& E. A. CANTORAL URIZA. 2016. La importancia ecológica de las algas en los ríos. Hidrobiológica 26: 1-8.

BOLTOVSKOY, A. 1999. Contribución al conocimiento de los dinoflagelados de la República Argentina. Tesis Doctoral. Facultad de Ciencias Naturales y Museo. Universidad Nacional de La Plata. Buenos Aires. Argentina, $117 \mathrm{pp}$.

BURNS, A. \& D. RYDER. 2001. Potential for biofilms as biological indicators in Australian riverine systems. Eco. Man. \& Res. 2: 53-63. https://doi.org/10.1046/ j.1442-8903.2001.00069.x.

CHAPMAN, D. \& V. KIMSTACH. 1992. The selection of water quality variables. En CHAPMAN, D. (ed.), Water quality assessments, pp. 51-119. Chapman y Hall, Londres, Inglaterra.

DE RUYTER VAN STEVENINCK, E. D., W. ADMIRAAL, L. BREEBAART, G. M. TUBBING \& B. VAN ZANTEN. 1992. Plankton in the River Rhine: structural and functional changes observed during downstream transport. J. Pl. Res. 14: 13511368.

DESIKACHARY, T. V. 1959. Cyanophyta. Ind. Counc. Agr. Res. New Delhi. India. 686 pp.

DOLBETH, M., M. A., PARDAL, A. I. LILLEBLO, U. AZEITEIRO \& J. C. MARQUES. 2003. Short- and long-term effects of eutrophication on the secondary production of an intertidal macrobenthic community. Mar. Biol. 10: 1133-1135. https://doi.org/10.1007/ s00227-003-1133-5.
ECHAZU, D. M. 2012. Biodiversidad de Diatomeas en Humedales del Sur de la provincia de Santa Cruz, Argentina. Tesis Doctoral. Facultad de Ciencias Exactas y Naturales. Universidad de Buenos Aires. Argentina. $354 \mathrm{pp}$.

FERNÁNDEZ, D. S. 2012. Estudio Geoquímico ambiental de la cuenca del río Colorado, Provincia de Tucumán, Argentina. Tesis Doctoral. Facultad de Ciencias Naturales e IML. Universidad Nacional de Tucumán, Tucumán. Argentina. 218 pp.

FERNÁNDEZ, R. 1981. Geología del extremo sur de la Sierra de San Javier y Potrero de las Tablas. Seminario de Grado. Facultad de Ciencias Naturales e IML. Universidad Nacional de Tucumán. Tucumán. Argentina. 299 pp.

FONTÚRBEL, F. 2005. Indicadores fisicoquímicos y biológicos del proceso de eutrofización del Lago Titikaka (Bolivia). Ecología Aplicada [en línea]. http://www.scielo.org.pe/pdf/ecol/v4n1-2/ a18v4n1-2.pdf.

FRÉMY, P. 1930. Les Myxophycées de l'Afrique équatoriale française. Archives de Botanique, Mémoires 3 (2): 1-508.

GEITLER, L. 1932. Cyanophyceae. En: Rabenhorst's Kryptogamenflora 14. 1196 pp. Akad. Verlag Ed., Leipzig, Alemania.

GERMAIN, H. 1981. Flore des diatomées -Diatomophycées- eaux douces et saumâtres du Massif Armoricain et des contrées voisines d'Europe occidentale Collection "Faunes et Flores Actuelles". Société Nouvelle des Editions Boubée, Paris. 444 pp.

GIORGI, A., M. BANCHERO, S. RIVELLI, O. CLARENSIO \& W. CUEVAS. 1999. Algunas variables indicativas de la calidad del agua del tramo medio del Río Luján. Memorias. VII Jornadas Pampeanas de Ciencias Naturales. COPROCNA. La Pampa, Argentina. 1 y 2 de junio, 2016. Pp. 155-162.

GONZÁLEZ, J. \& E. DOMÍNGUEZ. 1994. Efectos de los efluentes de una planta elaboradora de papel sobre la calidad del agua y composición biótica en el Arroyo Calimayo (Tucumán-Argentina). Ser. Conserv. Nat. 8: 3-12.

GONZÁLEZ ACHEM, A. L., C. SEELIGMANN \& M. ALDERETE. 2014. Variaciones espacio-temporales de la flora diatomológica en laguna de los Pozuelos (Jujuy, Argentina). Bol. Soc. Argent. Bot. 49: $177-$ 193.

GUIDO, E. \& P. SESMA. 2014. Geografía Física. En MOYANO, S., M. E. PUCHULU, D. S. FERNÁNDEZ, M. E. VIDES, S. NIEVA \& G. ACEÑOLAZA (eds.), Geología de Tucumán, pp. 2948. Colegio de Graduados en Ciencias Geológicas de Tucumán, Tucumán, Argentina.

HOLM-HANSEN, O. 1978. Chlorophyll a determination: improvements in methodology. Oikos A Journal of Ecology 30: 438-447. 
HUBER-PESTALOZZI, G. 1955. Das Phytoplankton des Süßwassers. Systematik und Biologie. Die Binnengewässer Bd. XVI, 7.

HUTSON, D. \& T. ROBERTS. 1990. Environmental fate of pesticides v. 7. Progression Pesticide Biochemistry and toxicology. John Wiley y Sons. 286 pp.

IRAM. 1996. Instituto Argentino de Normalización y Certificación - Norma IRAM 29012-2: calidad del medio ambiente: agua: muestreo: directivas generales sobre técnicas. Buenos Aires. 23 pp.

IRAM. 1998. Norma IRAM 29012 del Instituto Argentino de Normalización y Certificación - 3: calidad ambiental: calidad del agua: muestreo: guía para la preservación y manipulación de las muestras. Buenos Aires. 34 pp.

IRAM. 2005. Norma IRAM 301/ISO17025 del Instituto Argentino de Normalización y Certificación: requisitos generales para la competencia de los laboratorios de ensayo y de calibración. Buenos Aires. 37 pp.

ISASMENDI, S. C. 2016. Estudio del fitoplancton y epiliton de ríos de Tafi del Valle (Tucumán, Argentina). Tesis Doctoral. Facultad de Ciencias Naturales e IML. Universidad Nacional de Tucumán, Argentina. 307 pp.

ISASMENDI, S. C., C. SEELIGMANN, S. MARTÍNEZ DE MARCO \& B. TRACANNA. 2002. Flora diatomológica de un canal de descarga de desechos mineros (Tucumán-Argentina). Bol. Soc. Argent. Bot. 37: 41-49.

KARRASH, B., O. PARRA, H. CID, M. MEHRENS, P. PACHECO, R. URRUTIA, C. VALDOVINOS \& C. ZAROR. 2006. Effects of pulp and paper mill effluents on the microplankton and microbial self-purification capabilities of the Biobío River, Chile. Sc. Total Environ. 359: 194-208. https://doi. org/10.1016/j.scitotenv.2005.03.029.

KOMÁREK, J. \& K. ANAGNOSTIDIS. 2005. Cyanoprokaryota, Teil: Oscillatoriales. Susswasserflora von Mitteleuropa. Band 19/2. Spektrum Akademischer Verlag, Berlin. 759 pp.

KOMÁRKOVÁ-LEGNEROVÁ, J. 1969. The Systematics and Ontogenesis of the genera Ankistrodesmus Corda and Monoraphidium gen. nov. En FOTT, B. (ed.), Studies in Phycology, pp. 75-122. Academia, Praha.

KRAMMER, K. \& H. LANGE-BERTALOT. 1986. Bacillariophyceae 1. Teil: Naviculaceae. En: ETTL, H., J. GERLOFF, H. HEYNIG \& D. MOLLENHAUER (eds.). Süsswasserflora von Mitteleuropa, Veb Gustav Fischer Verlag, Jena, Alemania. $876 \mathrm{pp}$.

KRAMMER, K. \& H. LANGE-BERTALOT. 1988. Bacillariophyceae. 2. Teil: Bacillariaceae, Epithemiaceae, Surirellaceae. En ETTL H., J. GERLOFF, H. HEYNIG \& D. MOLLENHAUER (eds.). Süsswasserflora von Mitteleuropa, Band 2/2. Gustav Fischer Verlag, Stuttgart, New York. 596 pp.
KRAMMER, K. \& H. LANGE-BERTALOT. 1991. Bacillariophyceae. 3. Teil: Centrales, Fragilariaceae, Eunotiaceae. En ETTL, H., GERLOFF, J., HEYNIG, H. \& MOLLENHAUER, D. (eds.), Süsswasserflora von Mitteleuropa, Band 2/3. Gustav Fischer Verlag: Stuttgart, Jena. $576 \mathrm{pp}$.

KRAMMER, K. \& H. LANGE-BERTALOT. 2004. Bacillariophyceae. 4. Teil: Achnanthaceae. Kritische Ergänzungen zu Achnanthes s. 1. Navicula s. str., Gomphonema. En ETTL H., G. GÄRTNER, J. GERLOFF, H. HEYNIG \& D. MOLLENHAUER (eds.). Süsswasserflora von Mitteleuropa, Spektrum Akademischer Verlag, Heidelberg, Berlín, Alemania. 468 pp.

KRIEGER, W. 1937. Die Desmidiaceen Europas mit Berücksichtigung der aussereuropäischen Arten. I. En Rabenhorst's, Kriptogamen-Flora von Deutschland, Österreich und der Schweiz 13, 1: 1-712.

LEGENDRE P. \& L. LEGENDRE. 2000. Numerical Ecology. Second English Edition. Elsevier Science B. V. Montreal, Canada.

LELAND, H. V., L. R. BROWN \& D. K. MUELLER. 2001. Distribution of algae in the San Joaquin River, California, in relation to nutrient supply, and other environmental factors. Fresh. Biol. 46: 1139-1167. https://doi.org/10.1046/j.1365-2427.2001.00740.x.

LEVKOV, Z. 2009. Diatoms of Europa 5. Amphora sensu lato. A. R. G. Gantner Verlag K. G. Alemania.

LEVKOV, Z., D. METZELTIN \& A. PAVLOV. 2013. Diatoms of Europe of the European Inland waters and comparable habitats. Luticola and Luticolopsis. A.R.G. Gantner Verlag. Alemania.

LICURSI, M. \& N. GÓMEZ. 2013. Short-term toxicity of hexavalent-chromium to epipsammic diatoms of a microtidal estuary (Río de la Plata): Responses from the individual cell to the community structure. Aquatic Toxicology 134-135: 82-91.

LOEZ, C. 1995. Determinación de la clorofila $a$. En LOPRETTO, E. C. \& G. TELL (eds.), Ecosistemas de aguas continentales. Metodologías para su estudio. Pp. 263-269. Ed. Sur, La Plata, Buenos Aires, Argentina.

LUQUE, M. E. \& A. L. MARTÍNEZ de FABRICIUS. 2003. Distribución temporal del fitoplancton y epiliton en el río Piedra Blanca (Córdoba, Argentina). Limnetica 22: 19-34.

MAIDANA, N. I. \& C. SEELIGMANN. 2006. Diatomeas (Bacillariophyceae) de ambientes acuáticos de altura de la provincia de Catamarca, Argentina II. Bol. Soc. Argent. Bot. 41: 1-13.

MAIDANA, N. I. \& C. SEELIGMANN. 2015. Diatomeas (Bacillariophyceae) en humedales de altura de la Provincia de Catamarca (Argentina) III. Bol. Soc. Argent. Bot. 50: 447-466.

MAIDANA, N. I., C. SEELIGMANN \& M. R. MORALES. 2008. Diatomeas (Bacillariophyceae) de humedales de altura de la Provincia de Jujuy, Argentina. Bol. Soc. Argent. Bot. 43: 1-17. 
MAIDANA, N. I., C. SEELIGMANN \& M. R. MORALES. 2011. El género Navicula sensu stricto (Bacillariophyceae) en humedales de altura de Jujuy, Argentina. Bol. Soc. Argent. Bot. 46: 13-29.

MAGURRAN, A. E. 2004. Measuring biological diversity. Blackwell Publishing, Oxford, Reino Unido. 256 pp.

MARGALEF, R. 1997. Our Biosphere. Excellence in Ecology, Oldendorf/Luhe, Alemania.

MARTÍNEZ de FABRICIUS, A. L. 1996. Bacillariophyceae del Río Cuarto, Provincia de Córdoba. Argentina. Tesis Doctoral. Facultad de Ciencias Naturales y Museo. Universidad Nacional de La Plata, Buenos Aires. Argentina. 290 pp.

MARTÍNEZ de FABRICIUS, A. L., M. E. LUQUE, D. LOMBARDO \& M. D. NOVOA. 2010. Phytoplankton from Suco and La Felipa lakes, Córdoba, Argentina. [en línea]. http://www.alihuen. org.ar/algas-ficologia/algas-del-sur-de-cordoba-2. html.

MERCADO, L. M. 2001. Estructura del fitoplancton de seis sistemas lóticos de la provincia de Buenos Aires (Argentina). Rev. del Mus. de Cs. Nat. Nueva Serie. 3: 21-31.

METZELTIN, D., H. LANGE-BERTALOT \& F. GARCÍA RODRÍGUEZ. 2005. Diatoms of Uruguay. Compared with other taxa from South América and elsewhere. A. R. G. Gantner Verlag, Königstein, Alemania.

MEYBECK, M. 2003. Global analysis of river systems: from Earth system controls to Anthropocene syndromes. Philos. T. Roy. Soc. B. 358: 1935-1955. https://doi.org/10.1098/rstb.2003.1379.

MIQUELARENA, A. M., R. C. MENNI \& H. L. LÓPEZ. 1990. Ichthiological and limnological observations on the Salí river basin (Tucumán, Argentina). Ichthyol. Explor. Freshwaters 1: 269-276.

MIRANDE, V. 2001. Dinámica del fitoplancton del río Gastona (Tucumán, Argentina) en relación a la calidad de sus aguas. Tesis Doctoral. Facultad de Ciencias Naturales e IML. Universidad Nacional de Tucumán, Tucumán. Argentina. 271 pp.

MIRANDE, V. 2006. Riqueza del Fitoplancton del Arroyo Calimayo (Tucumán. Argentina). Lilloa 43: 61-86.

O'FARRELL, I., R. LOMBARDO, P. DE TEZANOS PINTO \& C. LOEZ. 2002. The assessment of water quality in the Lower Lujan River (Buenos Aires, Argentina): phytoplankton and algal bioassays. Environ. Pollut. 120: 207-218. https://doi. org/10.1016/S0269-7491(02)00136-7.

PADILLA TORRES, S. I., M. C. APELLA \& M. del V. HIDALGO. 1996. Ríos de la provincia de Tucumán. Conductividad y Clasificación. Serie Monográfica y Didáctica 32: 2-15.

PANTLE, R. \& H. BUCK. 1955. Die biologische Überwachung der Gewässer und die Darstellung der Egerbnisse. Gas- und Wasserfach. 96: 604-607.
PEIRCE, J. J., R. F. WEINER \& P. A. VESILIND. 1998. Environmental pollution and control, 4th ed. EUA.

PIELOU, E. C. 1966. The measurements of diversity in different types of biological collections. J. Theor. Biol. 13: 131-144. https://doi.org/10.1016/00225193(66)90013-0.

PIZARRO, H. \& M. ALEMANNI. 2005. Variables fisicoquímicas del agua y su influencia en la biomasa del perifiton en un tramo inferior del Río Luján (Provincia de Buenos Aires). Ecol. Austral 15: 73-88.

PLATAROTI, M. C. 2010. Caracterización de la calidad del agua de una sección del río Luján: Efectos sobre el fitoplancton. Tesina de Licenciatura. Universidad de Buenos Aires, Buenos Aires. Argentina. 64 pp.

PRESCOTT, G. W. 1961. Algae of Western Great Lakes Area. W. M. C. Brown Company Publishers, Dubuque, Iowa, EUA. 990 pp.

PRESCOTT, G. W., H. T. CROASDALE \& W. C. VINYARD. 1972. North American Flora, Desmidiales I: Saccodermae, Mesotaeniaceae. New York Botanical Garden, New York, EUA. 82 pp.

PUERTO RODRÍGUEZ, S. P. \& A. PIMENTEL SUÁREZ. 2006. Determinación de índices y planteamiento de objetivos de calidad del agua para las cuencas de segundo orden de la jurisdicción de La Car - Cundinamarca. Tesis de Ingeniería. Universidad de La Salle, Facultad de Ingeniería Ambiental y Sanitaria. Bogotá. Colombia. 164 pp.

R Development Core Team. 2005. R: a language and environment for statistical computing. $\mathrm{R}$ Foundation for Statistical Computing, Vienna, Austria.

RAMÍREZ GONZÁLEZ, A. \& G. VIÑA VIZCAÍNO. 1998. Limnología Colombiana: Aportes a su conocimiento y estadísticas de análisis. Editorial Panamericana. Bogotá, Colombia. 292 pp.

RODIER, J. 1990. Análisis de las Aguas. Ediciones Omega, Barcelona. España.

RODRÍGUEZ, C., M. MANCINI, C. PROSPERI, A. WEYERS, G. ALCANTÚ \& S. FERRERO. 2002. Variaciones estacionales de la calidad del agua del río Chocancharava (Río Cuarto), Córdoba, Argentina. Ecol. Austral 12: 65-72.

ROS, J. 1979. Prácticas de Ecología. Ed. Omega. Barcelona.

SABATER, S. \& F. SABATER. 1987. Comunidades de diatomeas en localidades altamente contaminadas del Río Ter (Cataluña, NE España). Limnetica 3: 103-109.

SALUSSO, M. \& L. MORAÑA. 2002. Comparación de índices bióticos utilizados en el monitoreo de dos sistemas lóticos del Noroeste Argentino. Rev. Biol. Trop. 50: 327-336.

SEELIGMANN, C. T. 2000. Nuevas citas de Bacillariophyceae para el Noroeste Argentino. Bol. Soc. Argent. Bot. 34: 165-169. 
SEELIGMANN, C. \& N. MAIDANA. 2003. Diatomeas (Bacillariophyceae) de ambientes acuáticos de altura de la provincia de Catamarca (Argentina). Bol. Soc. Argent. Bot. 38: 39-50.

SEELIGMANN, C. \& N. I. MAIDANA. 2013. ¿Existen especies de Navicula (Bacillariophyta) exclusivas de ambientes de alta montaña en Argentina? Bol. Soc. Argent. Bot. 48: 421-433.

SEELIGMANN, C., B. C. TRACANNA, S. N. MARTÍNEZ DE MARCO \& S. ISASMENDI. 2001. Algas fitoplanctónicas en la evaluación de la calidad del agua de sistemas lóticos en el Noroeste Argentino. Limnetica 20: 123-133.

SOSA, M., M. NOVOA \& A. L. MARTÍNEZ de FABRICIUS. 2011. Ficoflora de la Cuenca endorreica fluviolacustre Chucul (Córdoba, Argentina). Biológicas 13: 14-23.

STARMACH, K. 1966. Cyanophyta, Sinice Glaucophyta-Glaukofity. Woolma. Polski. 2. Pols. Ak. Inst. Bot.

TABOADA, M. Á., S. N. MARTÍNEZ DE MARCO \& B. C. TRACANNA. 2016. Biodiversidad epilítica de un arroyo subtropical del Noroeste Argentino. Lilloa 53: 122-132.

TELL, G. \& V. CONFORTI. 1986. Euglenophyta pigmentadas de la Argentina. Bibl. Phycol. Vol. 75.

TER BRAAK, C. J. \& P. ŠMILAUER. 1998. CANOCO reference manual and user's guide to CANOCO for Windows: software for canonical community ordination (Version 4). Ithaca, New York: Microcomputer Power.
TORO, J., J. P. J. SCHUSER KUROSAWA, E. ARRAYA \& M. CONTRERAS. 2003. Diagnóstico de la calidad del agua de sistemas lóticos utilizando diatomeas y macroinvertebrados bentónicos como bioindicadores, Río Maipo (Santiago de Chile). Sociedad Chilena de Ingeniería Hidráulica. IX Congreso Chileno de Ingeniería Hidráulica, p. 11.

TORRES SOLANO, D. P. 2008. Diagnóstico de la calidad del agua de la Microcuenca Sancotea. Ingeniería Ambiental Universidad Libre, pp. 55-66.

TRACANNA, B. C. 1982. Estudio taxonómico de las Chlorophyta de Tucumán (incluidas algunas consideraciones ecológicas). Opera Lilloana 32.

TRACANNA, B. C. 1985. Algas del Noroeste Argentino (excluyendo a las Diatomophyceae). Opera Lilloana 35.

UHERKOVICH, G. 1966. Die Scenedesmus-Arten Ungarns. Verlag der Ungarischen Akademie der Wissenschaften Akadémiai Kiadó, Budapest.

UTERMÖHL, H. 1958. Zur Vervollkommung der Quantitativen Phytoplankton Methodik. Mitt. Int. Verein. Limnol. 9: 1-38.

WESTERN, D. 2001. Human-modified ecosystems and future evolution. PNAS. 98: 5458-5465. https://doi. org/10.1073/pnas.101093598.

WHITTAKER, R. H. 1965. Dominance and diversity in land plant communities. Science 147: 250-260. https://doi.org/10.1126/science147.3655.250.

WHITTAKER, R. H. 1972. Evolution and measurement of species diversity. Taxon 21: 213-251. https://doi. org/10.2307/1218190.

Original recibido el 21 de abril de 2018; aceptado el 15 de agosto de 2018. 
